\title{
The Modeling and Stability Analysis of Humans Balancing an Inverted Pendulum
}

Yaohui Ding

Follow this and additional works at: https://researchrepository.wvu.edu/etd

\section{Recommended Citation}

Ding, Yaohui, "The Modeling and Stability Analysis of Humans Balancing an Inverted Pendulum" (2015). Graduate Theses, Dissertations, and Problem Reports. 5498.

https://researchrepository.wvu.edu/etd/5498

This Thesis is protected by copyright and/or related rights. It has been brought to you by the The Research Repository @ WVU with permission from the rights-holder(s). You are free to use this Thesis in any way that is permitted by the copyright and related rights legislation that applies to your use. For other uses you must obtain permission from the rights-holder(s) directly, unless additional rights are indicated by a Creative Commons license in the record and/ or on the work itself. This Thesis has been accepted for inclusion in WVU Graduate Theses, Dissertations, and Problem Reports collection by an authorized administrator of The Research Repository @ WVU. For more information, please contact researchrepository@mail.wvu.edu. 


\title{
The Modeling and Stability Analysis of Humans Balancing an Inverted Pendulum
}

\section{Yaohui Ding}

\author{
Thesis submitted to the \\ Benjamin M. Statler College of Engineering and Mineral Resources \\ at West Virginia University \\ in partial fulfillment of the requirements for the degree of \\ Master of Science \\ in \\ Mechanical Engineering
}

Dr. Yu Gu

Dr. Marvin H. Cheng

Dr. Jason Gross

Department of Mechanical and Aerospace Engineering

\author{
Morgantown, West Virginia
}

2015

Keywords: Inverted Pendulum, Modeling, Human Controller Estimation, Stability Analysis Copyright 2015, Yaohui Ding 


\section{ABSTRACT \\ The Modeling and Stability Analysis of Humans Balancing an Inverted Pendulum}

\section{Yaohui Ding}

The control of an inverted pendulum is a classical problem in dynamics and control theory. Without active control, the inverted pendulum by itself is inherently unstable, thus serving as an ideal platform for control algorithms design and testing. This study utilizes an inverted pendulum setup to investigate the characteristics of human manual control in executing a single-axial compensatory task. An inverted pendulum with sliding base on a single-axial rail was built for this purpose. Human subjects were asked to stabilize the pendulum by sliding the base on the rail. To mathematically quantify the characteristics of human manual control, a quasi-linear lead-lag with time delay model was chosen for the human operator. The mathematical model for the inverted pendulum was derived using the LaGrange's method. Using these two models, a simulation of the closed-loop human-inverted pendulum system was built in Matlab/Simulink. The stability conditions of the closed-loop system were derived by applying the RouthHurwitz stability criterion to the system. This completes the modeling and simulation of the process of humans balancing an inverted pendulum. The Matlab simulation serves as a validation tool in this study. The data of the human subject's input and the inverted pendulum's output generated from the simulation were used to estimate the parameters assumed in the mathematical model for the human operator. The estimation algorithm employed is a Kalman filter. Results show that the estimations do converge very quickly to the parameters set in the simulated human controller and can stabilize the inverted pendulum when fed back into the simulation. This verifies the plausibility of the mathematical structure for the human operator and the validity of the estimator. Experimentally, the pendulum's angle deflections from the vertical position and the human subjects' hand positions were recorded using a motion capture system called VICON. Using the same estimator developed for processing the simulation data, the collected experimental data were processed to estimate the parameters in the model for the human operator when the human operator actually carries out the task of balancing the inverted pendulum. The estimated parameters from the experimental data were then fed into the simulation model. The characteristics of the human operator were analyzed using the estimated parameters. 


\section{ACKNOWLEDGEMENTS}

I would like to take this opportunity to acknowledge the people who have mentored, helped and encouraged me along this journey.

First, I would like to thank my research advisor, Dr. Yu Gu, for his support, guidance and encouragement throughout this program. Dr. Gu, your support and guidance provided me with some great learning opportunities. Your patience and encouragement helped me overcame many difficult times within the last two years. I greatly appreciate your mentorship.

I would like to thank my committee members Dr. Marven Cheng, Dr. Jason Gross. Your discussions and feedback regarding this work were very helpful in improving its quality.

I would like to thank current and former students at the Interactive Robotics Laboratory at WVU, Tanmay Mandel, Kyle Lassak, Caleb Rice, Trevor Caplinger, Scott Harper, Alex Hypes, Jared Strader, and Lucas Behrens. Thank you for the help with my research. Your dedications and commitment to the research projects in our lab showed me what research is really about. I would also like to thank Dr. Srikanth Gururajan for his many wise and kind words.

I would also like to thank my family and some friends in China, for supporting and encouraging me throughout the program. Thank you for you love and support.

Finally, I would like to express my gratitude to the Almquist family, who has made my nearly two years' stay in Morgantown so much more enjoyable and memorable. Thank you for your kindness and hospitality.

This research was partially supported by NASA project NNX12AM56A. 


\section{Table of contents}

ABSTRACT

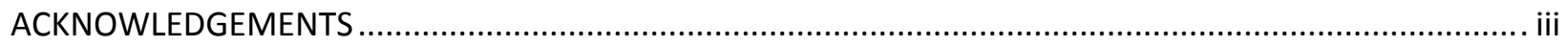

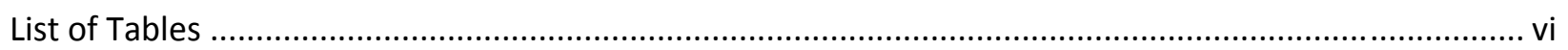

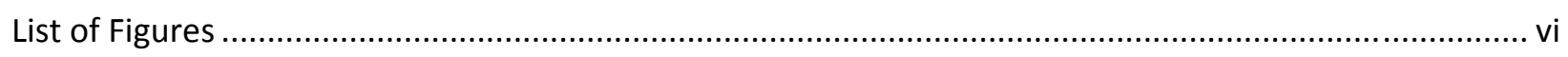

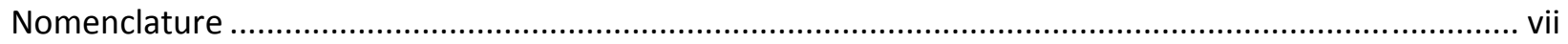

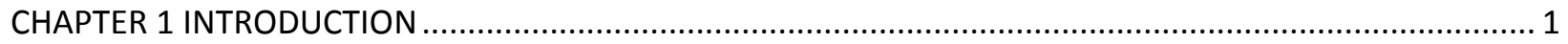

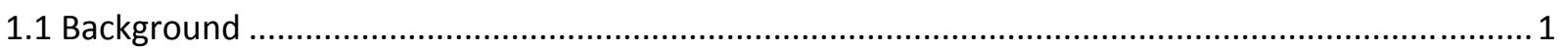

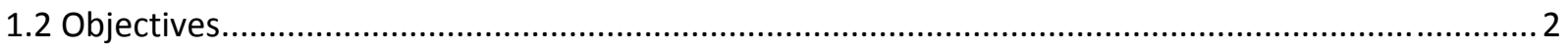

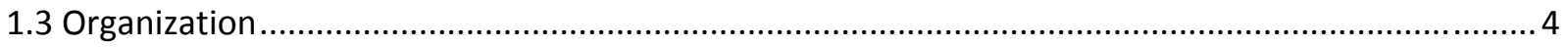

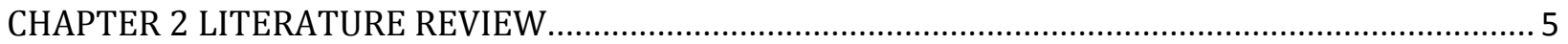

CHAPTER 3 EXPERIMENTAL SETUP AND DATA COLLECTION ................................................................ 7

3.1 Design of the Experimental Inverted Pendulum Setup in Solidworks ............................................. 7

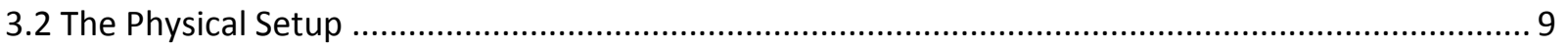

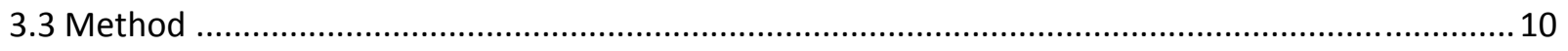

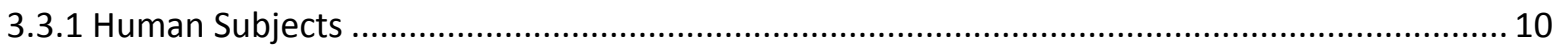

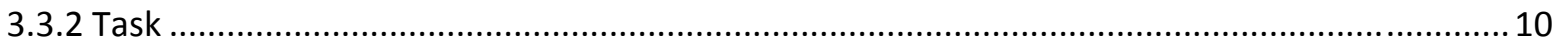

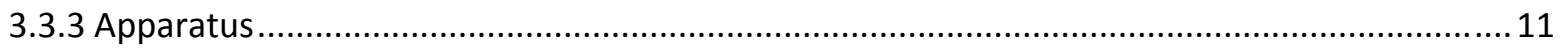

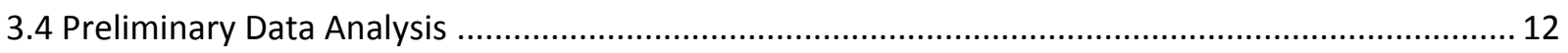

CHAPTER 4 MODELING OF THE HUMAN-INVERTED PENDULUM SYSTEM .......................................... 16

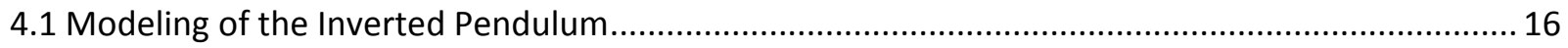

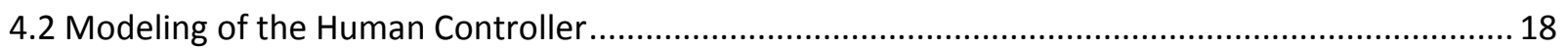

CHAPTER 5 STABILITY ANALYSIS OF THE HUMAN-INVERTED PENDULUM SYSTEM ..................................2

5.1 Stability Analysis of the Closed-loop Human-Inverted Pendulum System ...................................... 24

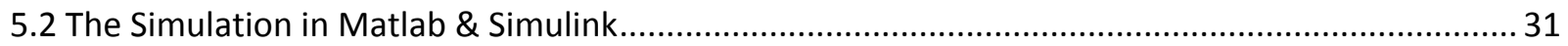

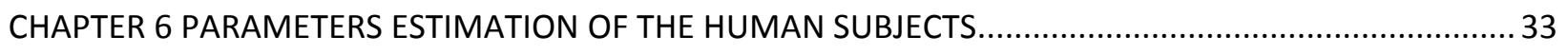

6.1 Estimation Algorithm Selection and Verification ....................................................................... 33

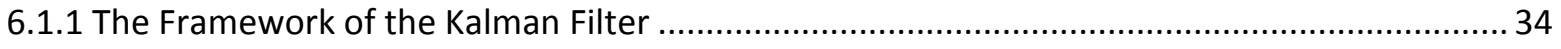

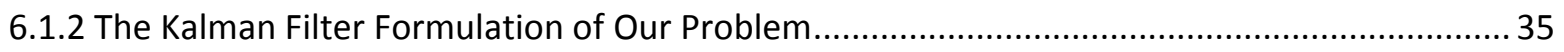

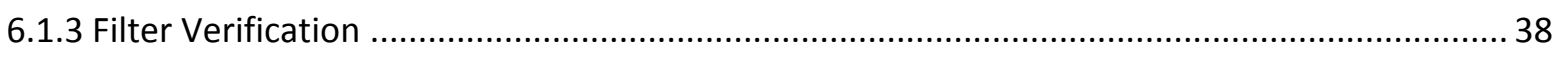

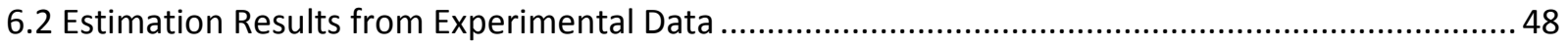

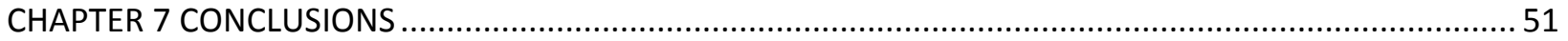




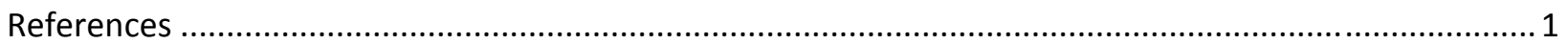

Appendix one Derivation of the EOM's of the Inverted Pendulum Setup.............................................. 1 


\section{List of Tables}

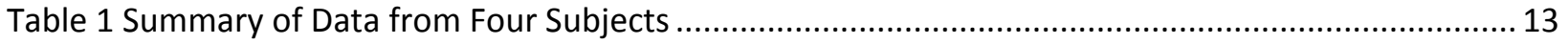

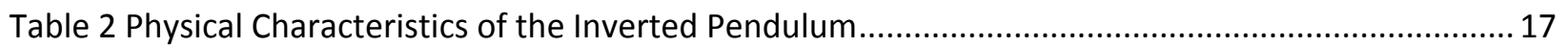

Table 3 Mean and standard deviation of delta k, delta T1, delta T2 for case 1 ....................................... 40

Table 4 Statistics of delta K, delta T1 and delta T2 for case 2 ........................................................... 42

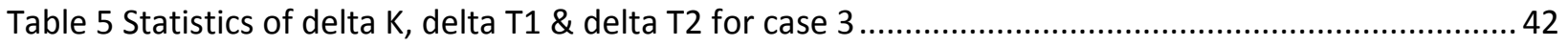

Table 6 Statistics of delta K, delta T1 \& delta T2 for case 4 ................................................................... 44

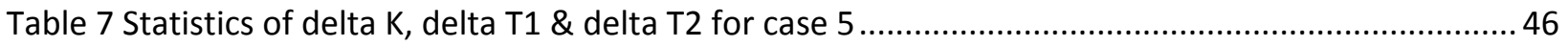

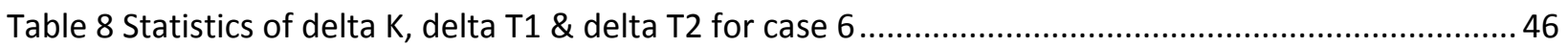

\section{List of Figures}

Figure 1 Schematic of an Inverted Pendulum Setup......................................................................... 1

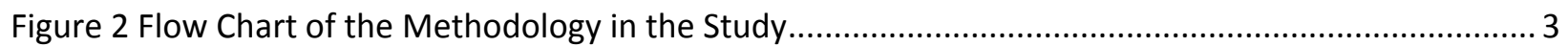

Figure 3 Solidworks Design of the Inverted Pendulum Setup ................................................................ 8

Figure 4 Exploded View of the Inverted Pendulum Setup Design ...................................................... 8

Figure 5 The Physical Experimental Inverted Pendulum Setup ............................................................. 9

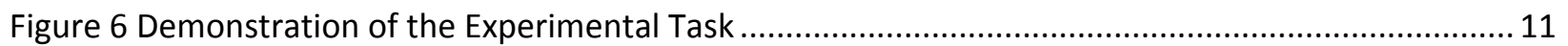

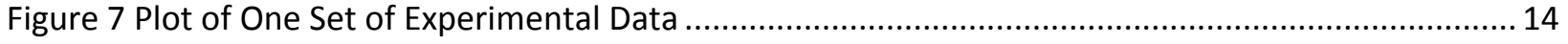

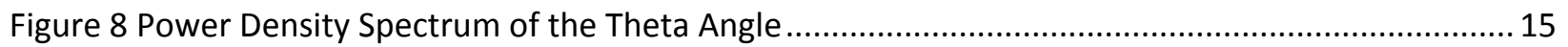

Figure 9 Power Density Spectrum of the Subject's Hand Movement.................................................... 15

Figure 10 Schematic of the Inverted Pendulum-Cart System ............................................................... 16

Figure 11 Schematic of the Progress of the Visual Signal in the Brain [28] ............................................ 19

Figure 12 Block diagram of the Closed-loop Human-inverted Pendulum .............................................. 24

Figure 13 Stability conditions of K, T1 \& T2 with different time delays ................................................... 30

Figure 14 Plots of the stable closed-loop human inverted pendulum system ......................................... 31

Figure 15 Matlab/Simulink Mode of the Human-Inverted Pendulum System ........................................ 32

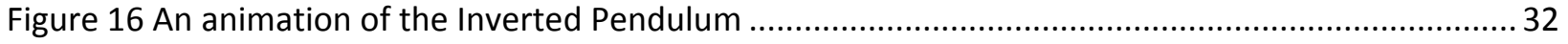

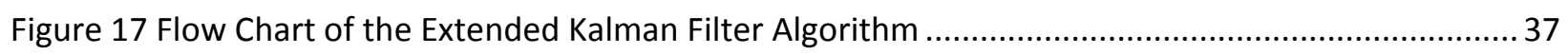

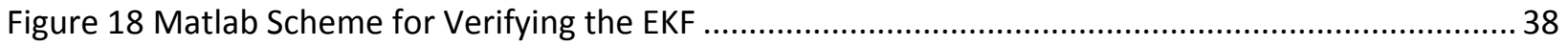

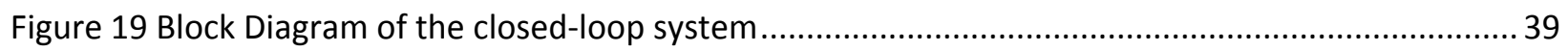

Figure 20 Plots of K, T1 \& T2 as R varies, without noise compensated ................................................... 40

Figure 21 Estimation of K, T1 \& T2 as R varies, with noise compensated .............................................. 41

Figure 22 Estimations of K, T1 \& T2 as R varies, with remnant noise.................................................... 43

Figure 23 Estimations of $\mathrm{K}, \mathrm{T} 1 \mathrm{\&}$ T2 as tao increases, with remnant and delay ......................................... 44

Figure 25 Estimations of $\mathrm{K}, \mathrm{T} 1 \mathrm{\&}$ T2 as delta tao increases, with remnant and delay ..............................45

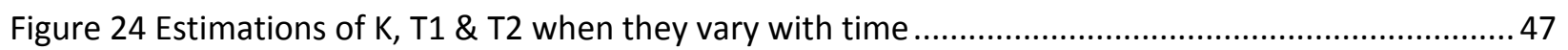

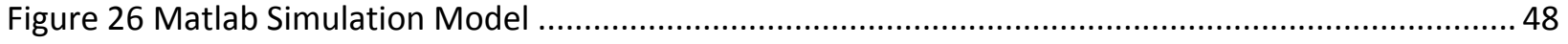

Figure 27 Estimations of $K, T 1$ \& T2 from experimental data, subject 1 ............................................... 49

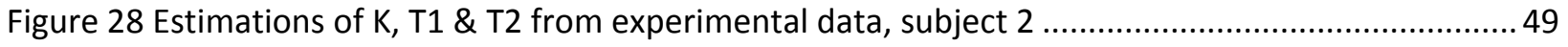

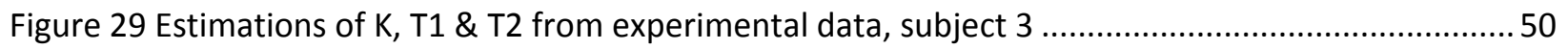




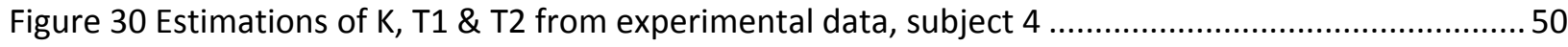

Figure 31 Schematic of the Inverted Pendulum-Cart System ................................................................ 1

\section{Nomenclature}

Acronyms

BIBO Bounded-Input Bounded-Output

CM Center of Mass

EKF Extended Kalman Filter

IRL Interactive Robotics Laboratory

LTI L Linear Time-Invariant

NNC Neural Network Controller

PID Proportional Integral Derivative

UKF Unscented Kalman Filter

WVU West Virginia University

English Symbols
A
State transition matrix
B Input matrix
e(s) Theta input to the human controller
E
Expectation operator
g
Earth's sea-level gravitational constant
H
Measurement matrix
।
Half of the length of the pendulum
$I_{m}$
Moment of inertia of the inverted pendulum
K
Kalman Gain Matrix
L1
Length of the rail
m
Mass of the pendulum 


$\begin{array}{ll}\text { M } & \text { Mass of the sliding base } \\ \mathbf{Q} & \text { State Error Covariance Matrix } \\ \mathbf{R} & \text { Assumed Process Noise Covariance Matrix } \\ \mathrm{S} & \text { Assumed Measurement Noise Covariance Matrix } \\ T_{1} & \text { Human Operator Lead Variable } \\ T_{2} & \text { Human Operator Lag Variable } \\ \mathrm{u} & \text { Human subject output } \\ \mathrm{x} & \text { Distance of the sliding base from the center of the rail } \\ Y_{p} & \text { Pilot transfer function } \\ Y_{c} & \text { Controlled element transfer function }\end{array}$

\section{Greek Symbols}

$\theta \quad$ Angular displacement of the pendulum from the vertical axis

$\mu \quad$ Mean

$\sigma \quad$ Standard deviation

$\tau_{1} \quad$ Half of the effective delay

$\tau_{e} \quad$ Effective delay

$\omega_{c} \quad$ Crossover frequency 


\section{CHAPTER 1 INTRODUCTION}

\subsection{Background}

An inverted pendulum is a pendulum with a pivot point at the bottom of it. The pivot point is often mounted on a cart that can slide on a single-axial rail (see Figure 1). The pendulum-cart system is inherently unstable, nonlinear, non-minimum phase and under-actuated, thus must be actively controlled in order for the pendulum to remain in the upright position. This can be achieved through many ways, such as by applying a torque at the pivot point, moving the pivot point horizontally using some feedback mechanisms, or even oscillating the pivot point vertically. The problem of balancing an inverted pendulum is a classical one in dynamics and control theory and is often used as a benchmark tool for testing and evaluating control algorithms, such as PID controllers, neural networks, fuzzy control, genetic algorithms etc.

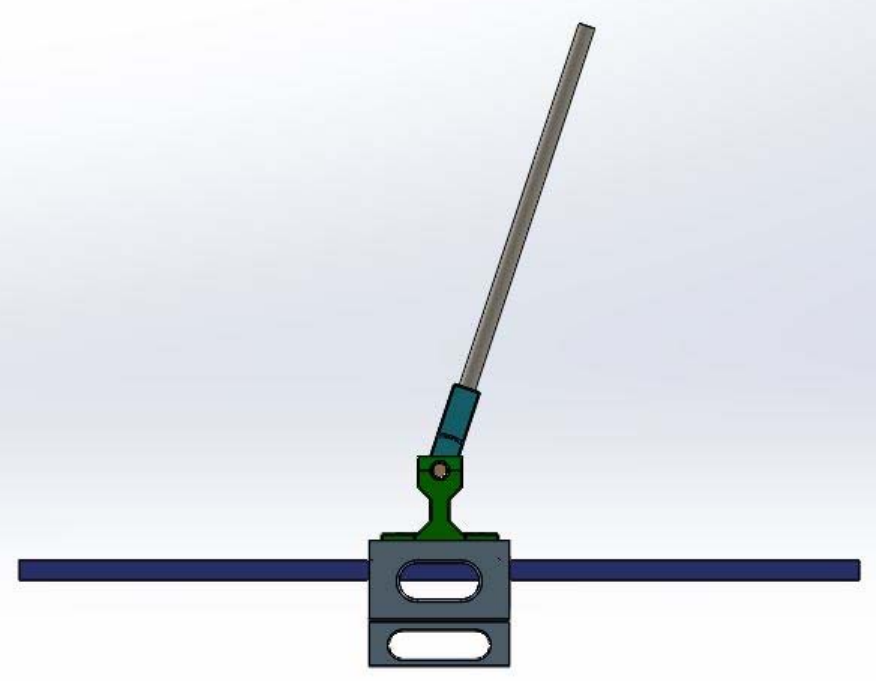

Figure 1 Schematic of an Inverted Pendulum Setup 


\subsection{Objectives}

The focus of this study is to investigate the characteristics of human manual control when executing a task of balancing an inverted pendulum on a single-axial rail. Suppose an automatic controller was designed for this task, the inverted pendulum should be able to stay in the upright position indefinitely as long as there are not any significant disturbances to the system. However, if the automatic controller was replaced by a human subject, falling of the inverted pendulum is almost inevitable no matter how skillful or agile the subject is. What is it then that leads to the falling of the inverted pendulum? Since the dynamics of the inverted pendulum does not really change in the course of the experiment, the human subjects must have undergone some changes which result in the falling of the pendulum. How do we quantify these changes? How might we predict the eventual falling of the inverted pendulum, maybe a few steps ahead of its occurring? These are the questions this study attempts to investigate and solve. To quantify the changes the human subjects undergo, a mathematical model of the human subjects is needed first. The next step is to estimate the values of the parameters in this model. To accomplish this, data from human subjects carrying out the experiment of balancing a real single-axial inverted pendulum has to be collected. Thus, an experimental inverted pendulum setup has to be constructed in the first place. Finally, using the parameters estimated from the experimental data, one can investigate the stability conditions of the human-inverted pendulum-cart system and hopefully to be able to predict the falling of the inverted pendulum. In summary, there are four major objectives in this study as listed below. In addition, the flow chart of the methodology used in the study is shown in the figure 2.

Objectives:

1. To build a physical inverted pendulum-cart system for human subjects to balance and for experimental data collection 
2. To model the inverted pendulum and the human operator and build a simulation of the human inverted pendulum-cart system in Matlab

3. To investigate the stability conditions of the human-inverted-pendulum-cart system

4. To estimate the parameters in the human operator mathematical model and use them to make predictions

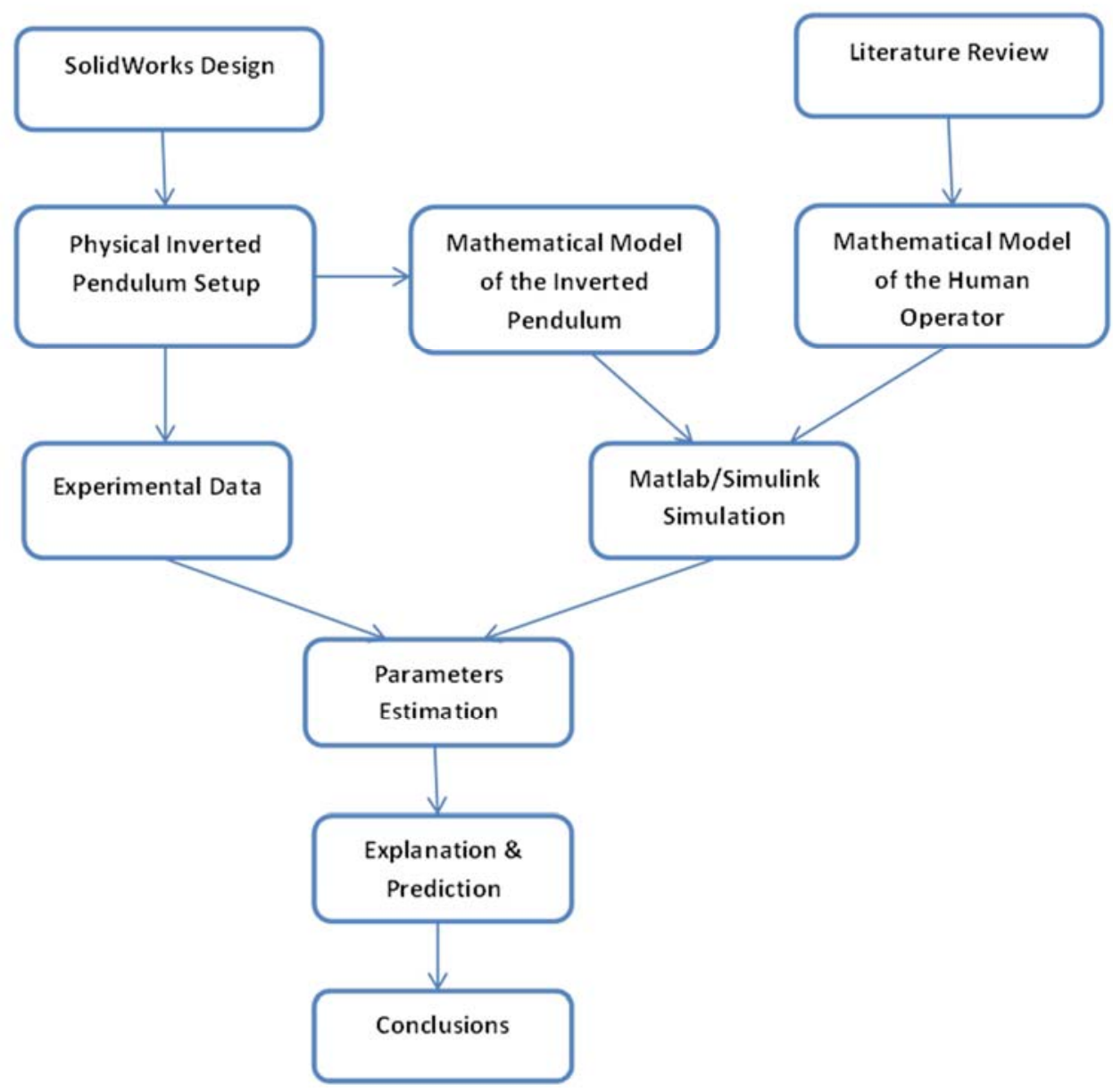

Figure 2 Flow Chart of the Methodology in the Study 


\subsection{Organization}

The rest of the thesis is organized as follows. Chapter 2 includes a literature review for this study. Chapter 3 describes the design of the inverted pendulum setup in Solidworks and the construction of the physical inverted pendulum. It also describes the data acquisition device, the experiments designed to obtain experimental data and some preliminary analysis of the data. Chapter 4 describes the process of modeling the inverted pendulum system and the human operator, in order to obtain a closed-loop mathematical model for the human-inverted pendulum-cart system. Using the mathematical model obtained, a simulation model is built in Simulink. Chapter 5 presents a stability analysis of the closedloop human-inverted pendulum system. Chapter 6 explains the data filtering algorithm used to estimate the values of the parameters in the mathematical model for the human operator and the estimation results. Finally, chapter 7 summarizes and concludes this work, with discussions regarding the future directions of this research topic. 


\section{CHAPTER 2 LITERATURE REVIEW}

The problem of stabilizing the pendulum in the upright position has attracted attentions from and been widely studied among control engineers, physicists, mathematicians and neuroscientists over the last few decades. First of all, the inverted pendulum serves as a benchmark tool for testing control algorithm. In 1963, Widrow et al. built an original adaptive neural net broom-balancer at Stanford University, to demonstrate the capability of the ADALINE (Adaptive Linear Neuron) in performing the task of optimal control of an unstable system[1]. The ADALINE network, a trainable system, observes the cart and the broom (pendulum) through visual inputs to obtain the relevant state information by dynamic scene analysis[2]. Simulation results show that, with adequate training sample, this simple adaptive neural network is capable of extracting the necessary state information from time sequence of crude visual images and using this information to balance the pendulum[3]. Many others have also taken interest in using the inverted pendulum-cart system to implement and test a variety of neural network controllers. Anderson [4] showed that it is possible to balance an inverted pendulum with no a priori knowledge of the dynamics of the system by implementing a two-layer neural network controller; Wieland [5] presented a way of using Genetic Algorithm to create recurrent neural networks to solve the pole balancing problem; Jung et al [6] used a wheel-driven mobile inverted pendulum platform to test the robustness of a neural network learning algorithm coupled with a PID controller. Besides of being a great tool in testing neural network control algorithm, the inverted pendulum setup has also been employed to test other advanced control algorithm such as fuzzy-logic controllers. Magana et al.[7] , used an inverted pendulum setup to evaluate the performance of a fuzzy-logic control system they designed. The fuzzy logic controller was able to keep the inverted pendulum in the upright position through visual feedback. Wang [8], used the inverted pendulum setup to validate that the adaptive fuzzy controller they designed could perform successful tracking with using any linguistic information and after incorporating some linguistic fuzzy rules into the controller, the adaptation speed became faster 
and the tracking error smaller. Mohamed et al.[9] , also presented an adaptive fuzzy-logic controller for controlling the inverted pendulum cart system as an under-actuated one. Swing up control of the inverted pendulum has also yield many fruitful results in the literature of control algorithm design [1013].

The applications of modeling and the stability study of the inverted pendulum, range from understanding human posture stability, to biped humanoid walking pattern generation, to modeling slender structures during earthquake and so forth. Kajita et al. did much of the pioneering research in employing the inverted pendulum model to solving problems such as, walking pattern generation for biped robots, walking stabilization for humanoids, biped locomotion on rugged terrain etc.[14-18]. Many other authors have also used varied versions of the inverted pendulum model to investigate the problem of biped robot walking. For example, Park [19] proposed a gravity compensated inverted pendulum model to generate biped locomotion patterns; Kuo [20] showed that the inverted pendulum model is better than the six determinants of gait model in representing the human walking dynamics. Based on the inverted pendulum model, Sugihara [21] proposed a zero moment point manipulation method for real-time humanoid motion generation. Gage [22] demonstrates the validity of using the inverted pendulum model to represent the kinematic and kinetic postural control in human quiet standing. Winter [23] reviewed the various versions of the inverted pendulum model used in assessing the human balance and posture control during standing and walking. Reeves [24] gained some interesting insights into the control and stabilization of the human spine from a series of stick balancing experiments on finger tips. Using the inverted pendulum model, Housner [25] did an analogous study of the rocking motion of tall and slender structures during earthquakes. 


\section{CHAPTER 3 EXPERIMENTAL SETUP AND DATA COLLECTION}

This chapter describes the design and construction of the inverted pendulum setup used in the experiment for data collection, the designed experiment, the data collection devices and some primary analysis of the obtained data.

\subsection{Design of the Experimental Inverted Pendulum Setup in Solidworks}

Before the physical inverted pendulum setup was constructed, a $3 \mathrm{~d}$ model of the setup was designed using Solidworks (see figure $3 \&$ figure 4). The design requirements include constraining the inverted pendulum's movement in a single plane, ensuring the pendulum can free rotate around the pivot point. Based on these requirements, the pendulum is designed to be rigidly attached to a rotating shaft which is mounted on the sliding basing through a pair of plain bearings. The rotating shaft ensures that the pendulum can freely rotate around the pivot point. The sliding based is design to be able to slide on a single axial rail with minimum frictions. A bracket which can be mounted to the sliding block is designed so that the human subject can use it to slide the base to balance the pendulum. As like the design of any other mechanisms, the process of designing the inverted pendulum setup was an iterative one. 


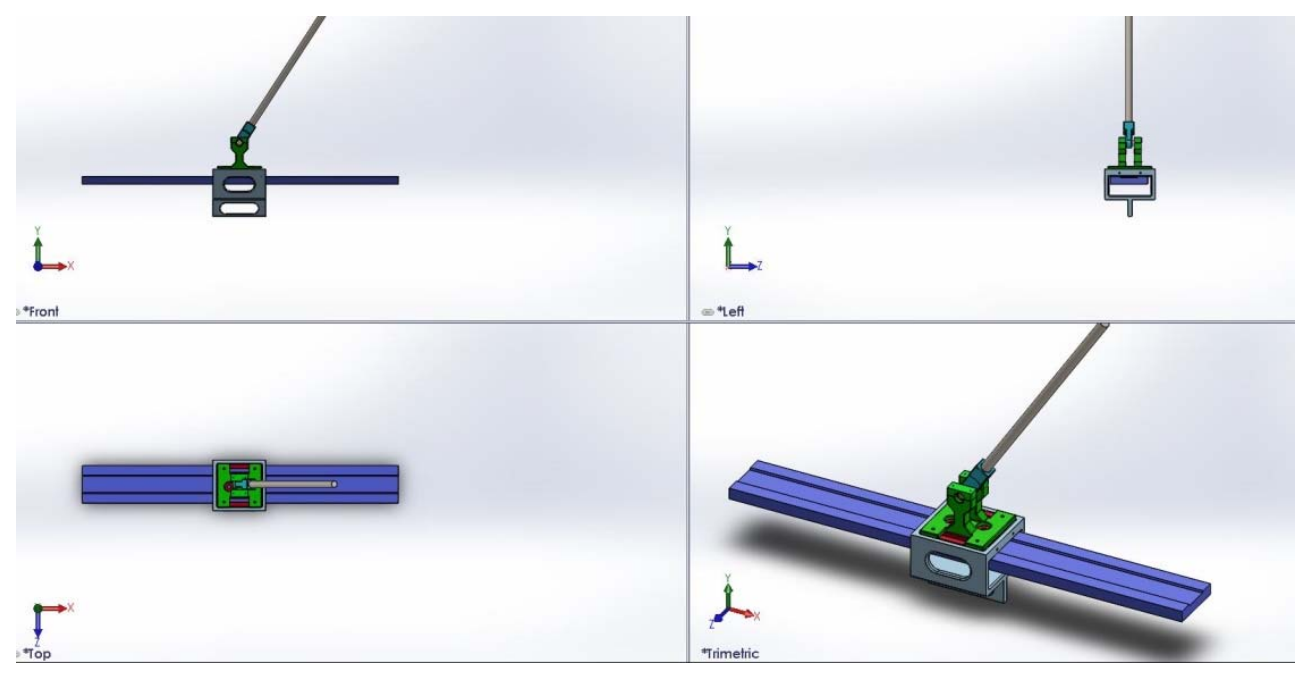

Figure 3 Solidworks Design of the Inverted Pendulum Setup

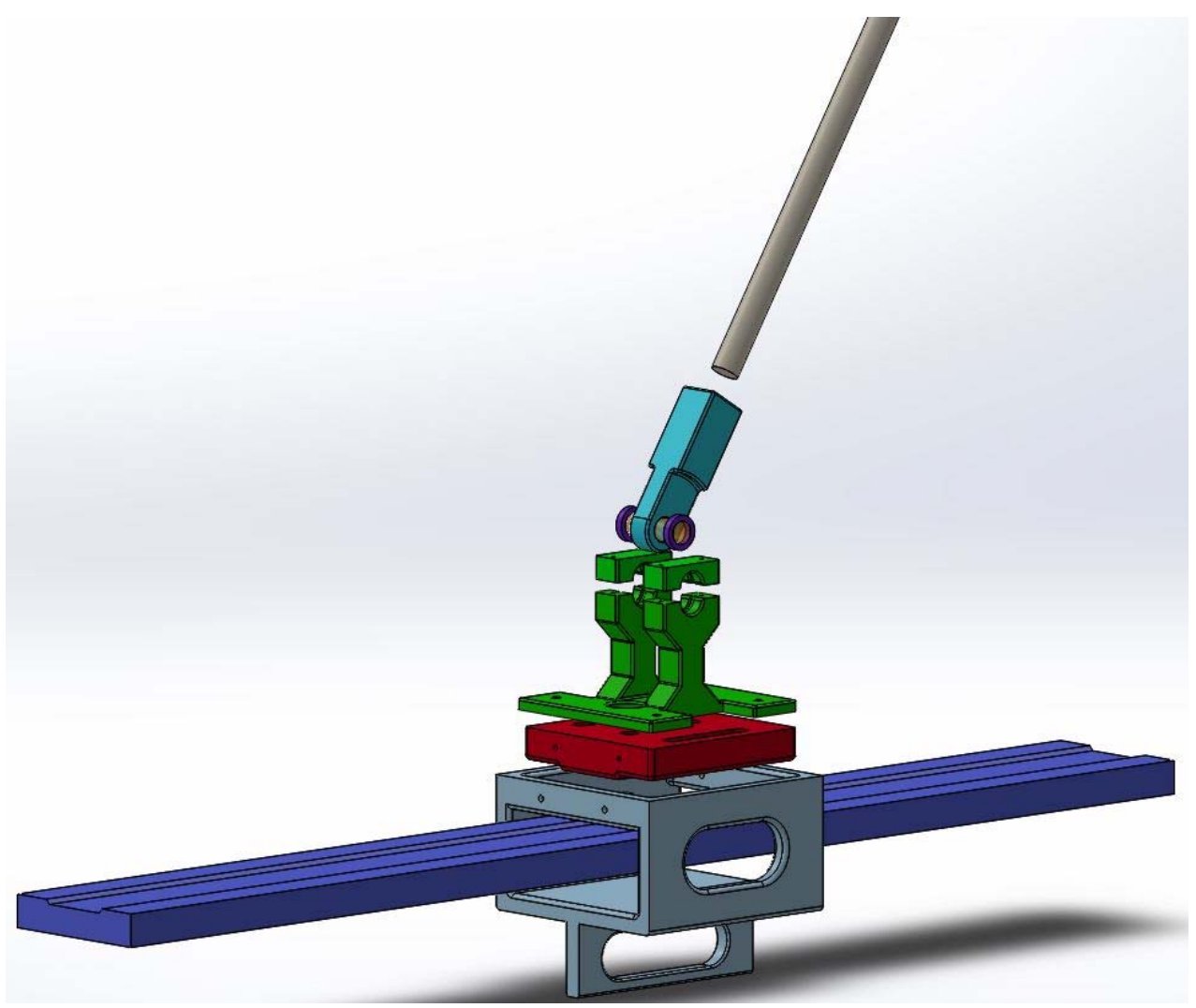

Figure 4 Exploded View of the Inverted Pendulum Setup Design

8 


\subsection{The Physical Setup}

A picture of the experimental setup is provided in figure 5 . The single axil rail is an off-the-shelf product.

It is 1.27 meters long and has a sliding block. The sliding block has the ability to adjust the tightness to reduce the sliding friction. The single axial rail is mounted on a pair of sawhorse legs. The height of the sawhosre is 0.711 meters. The pendulum is a piece of carbon fiber tube. Most other componnets are printed using a $3 d$ printer in the Interactive Robotics Laboratory (IRL) at West Virginia University. Constraints imposed by the physical world dictate that frictionless contact between the rail and the cart is impossible to achieve. But the friction is very small that it is reasonable to ignore the frictions between the sliding base and the rail, the pivoting joint and the contact surface.

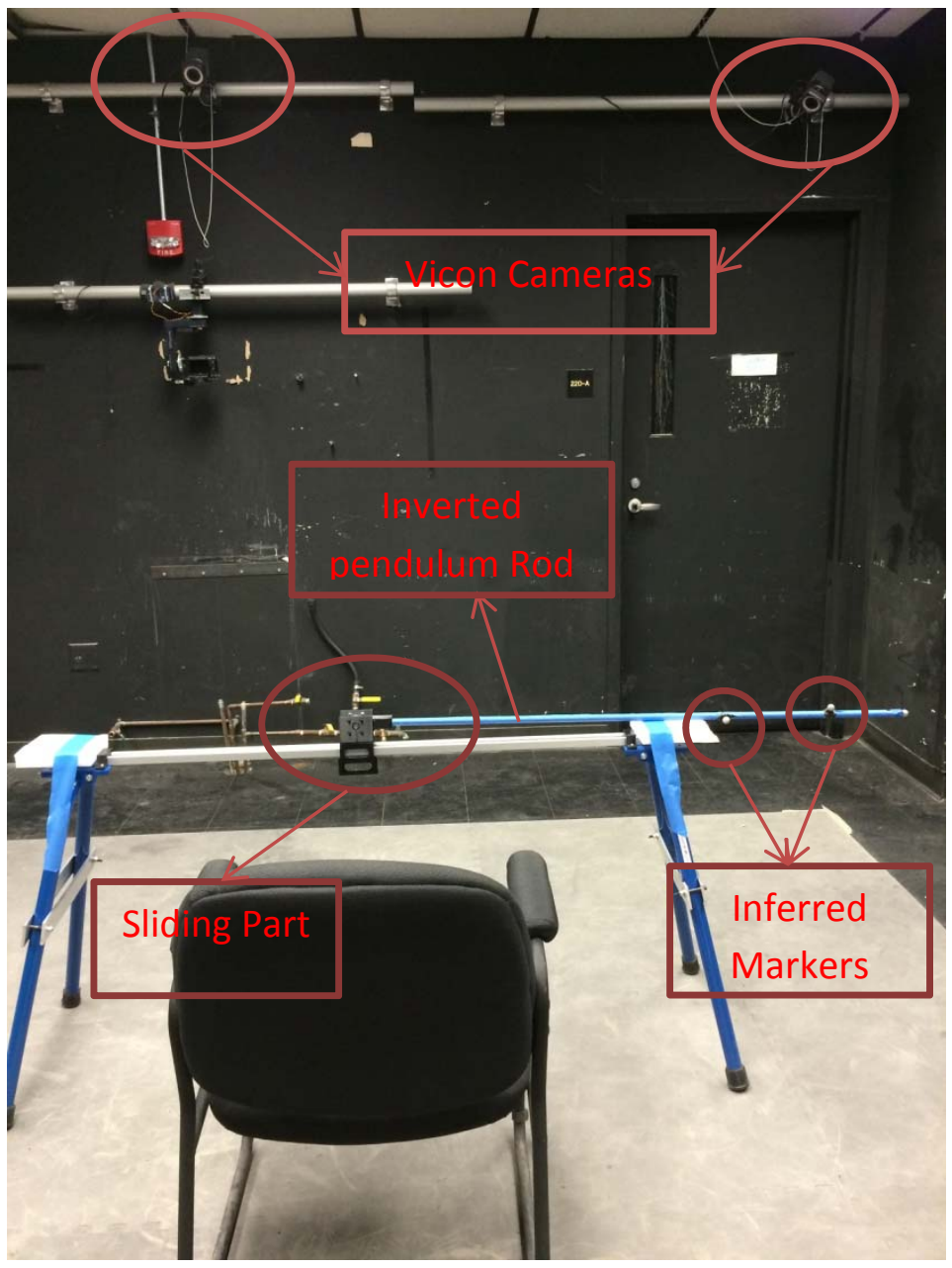

Figure 5 The Physical Experimental Inverted Pendulum Setup 


\subsection{Method}

This section describes the experiment carried out in the study and the data collection devices used in the experiment, participant information and preliminary analysis of the data collected.

\subsubsection{Human Subjects}

Four male students, age from 22 to 26, from West Virginia University with normal motor skills participated in this study. They all gave informed consent prior to their participation.

\subsubsection{Task}

Subjects were asked to keep the inverted pendulum in the upright position by sliding the base of the inverted pendulum. In the course of the experiment, the subject sits in an upright position in front of the inverted pendulum setup (as in figure 6). The starting position of the sliding base is near the center of the rail. The subjects were also requested to avoid the sliding base making contact with either end of the rail. The subjects were encouraged to pay their full attention to the task and keep the pendulum from falling for as long as possible. 


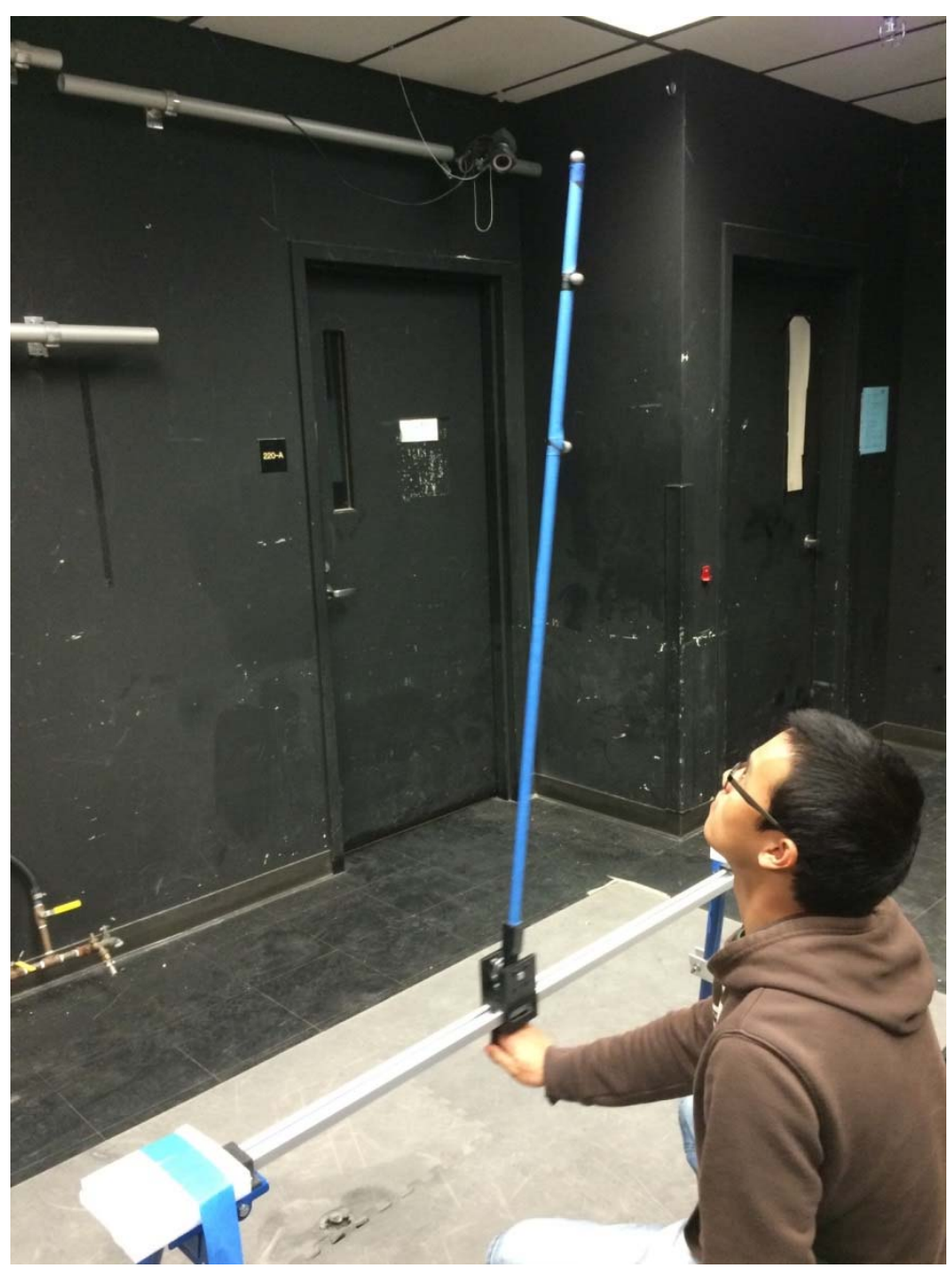

Figure 6 Demonstration of the Experimental Task

\subsubsection{Apparatus}

The angular displacement of the pendulum from the vertical position and the human subject's movement are the two variables measured in this experiment. IRL is equipped with a motion capture system called Vicon. The Vicon system consists of 8 Bonita B10 infrared cameras with 1 megapixels resolution and 250 maximum frame rates, a Tracker software system that can output data to Matlab with a low latency. The Vicon Tracker can track multiple objects. There are infrared markers attached to both the pendulum and the sliding base so their movements can be tracked by the Vicon system. 


\subsection{Preliminary Data Analysis}

Table 2 summarizes the data collected from four different subjects balancing the inverted pendulum. It includes the duration of the balance, the maximum and minimum angular displacements while the pendulum was in balance, the mean and standard deviation of the angular displacement, the maximum and minimum value of the human subjects' hand position, and the mean and standard deviation of the hand position. Figure 7 is the plots of the angular displacement theta and the human subjects' hand movement during one trial of experiment. Later in chapter 6 , these data will be used to estimate the parameters in the human subjects' mathematical model. Figure 8 and 9 show the power spectral density of the data set plotted in figure 7. The power spectrum shows the frequency components of a time series signal and how they are distributed. For a linear system, the power spectrum densities of the input and the output should have the same frequency components as a linear system does not generate new frequency components in the output signal given an input signal. A close examination of the power spectrum density of the angular displacement theta and the subjects' hand movements $\mathrm{x}$ shows that they are approximately the same in shapes. This information makes the assumption of linearizing the human subject's control behavior reasonable, which will be done in chapter 4 when the model of the human subject is obtained. Overall, these are just some preliminary analyses of the experimental data. In chapter 6, an estimator will be developed to process the data in order to estimate the parameters in the human subject's mathematical model. 


\begin{tabular}{|c|c|c|c|c|c|c|c|c|c|c|}
\hline \multirow[t]{2}{*}{$\begin{array}{l}\text { Sub } \\
\text { ject }\end{array}$} & \multirow[t]{2}{*}{$\begin{array}{l}\text { Trail } \\
\text { No. }\end{array}$} & \multirow[t]{2}{*}{$\begin{array}{c}\text { Tim } \\
\mathrm{e}\end{array}$} & \multicolumn{3}{|c|}{ Angle theta in radians } & \multirow[t]{2}{*}{$\sigma(\theta)$} & \multicolumn{3}{|c|}{$\begin{array}{c}\text { Hand position in } \\
\text { meters }\end{array}$} & \multirow[t]{2}{*}{$\sigma(x)$} \\
\hline & & & $\max$ & $\min$ & $\mu$ & & $\max$ & $\min$ & $\mu$ & \\
\hline \multirow{6}{*}{ A } & 1 & 33 & 0.2017 & -0.2790 & -0.0538 & 0.011 & 0.43 & - & 0.047 & 0.1 \\
\hline & & & & & & 31 & 51 & $\begin{array}{c}0.329 \\
3\end{array}$ & 0 & 699 \\
\hline & 2 & 34 & 0.1524 & -0.2753 & -0.0541 & 0.085 & 0.40 & - & 0.022 & 0.1 \\
\hline & & & & & & 7 & 18 & $\begin{array}{c}0.365 \\
4\end{array}$ & 7 & 272 \\
\hline & 3 & 23 & 0.1736 & -0.2971 & -0.0543 & 0.108 & 0.39 & - & 0.048 & $\begin{array}{l}0.1 \\
70\end{array}$ \\
\hline & & & & & & & & $\begin{array}{c}0.313 \\
1\end{array}$ & & \\
\hline \multirow{9}{*}{ B } & 1 & 25 & 0.1812 & -0.2847 & -0.0561 & 0.106 & 0.48 & - & 0.060 & 0.1 \\
\hline & & & & & & 9 & 31 & 0.285 & 2 & 482 \\
\hline & 2 & 20 & 0 & 0 & n & & & 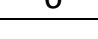 & & \\
\hline & & & ט & 0.2001 & כגנט. & .074 & ר.2 & 5 & 0017 & 0.1 \\
\hline & & & & & & & & 0.399 & 0.012 & \\
\hline & & & & & & & & & 1 & \\
\hline & 3 & 30 & 0.0940 & -0.2056 & -0.0555 & 0.060 & 0.41 & - & 0.087 & 0.1 \\
\hline & & & & & & 3 & 07 & 0.398 & 9 & 851 \\
\hline & & & & & & & & 5 & & \\
\hline \multirow{8}{*}{ C } & 1 & 28 & 0.1165 & -0.2194 & -0.0553 & 0.067 & 0.36 & - & 0.021 & 0.1 \\
\hline & & & & & & 5 & 60 & 0.342 & 9 & 394 \\
\hline & & & & & & & & 9 & & \\
\hline & 2 & 38 & 0.0960 & -0.2037 & -0.0538 & 0.041 & 0.46 & - & 0.035 & 0.1 \\
\hline & & & & & & 7 & 49 & 0.153 & 4 & 114 \\
\hline & & & & & & & & 0 & & \\
\hline & 3 & 25 & 0.2239 & -0.2187 & - & 0.072 & 0.41 & - & 0.012 & 0.1 \\
\hline & & & & & $\begin{array}{c}0.0483 \\
2\end{array}$ & 3 & 25 & $\begin{array}{c}0.356 \\
4\end{array}$ & 5 & 256 \\
\hline \multirow{9}{*}{ D } & 1 & 12 & 0.1932 & -0.2436 & -0.0474 & 0.067 & 0.44 & - & 0.032 & 0.1 \\
\hline & & & & & & 9 & 39 & 0.251 & 7 & 752 \\
\hline & & & & & & & & 9 & & \\
\hline & 2 & 11 & 0.1619 & -0.1943 & -0.0372 & 0.088 & 0.52 & - & 0.038 & 0.2 \\
\hline & & & & & & 4 & 89 & 0.356 & 3 & 081 \\
\hline & 3 & 14 & 0.1563 & -0.2004 & -0.0487 & 0.057 & 0.11 & & - & 0.1 \\
\hline & & & & & & 6 & 73 & - & 0.069 & 188 \\
\hline & & & & & & & & 0.285 & 7 & \\
\hline & & & & & & & & 0 & & \\
\hline
\end{tabular}

Table 1 Summary of Data from Four Subjects 


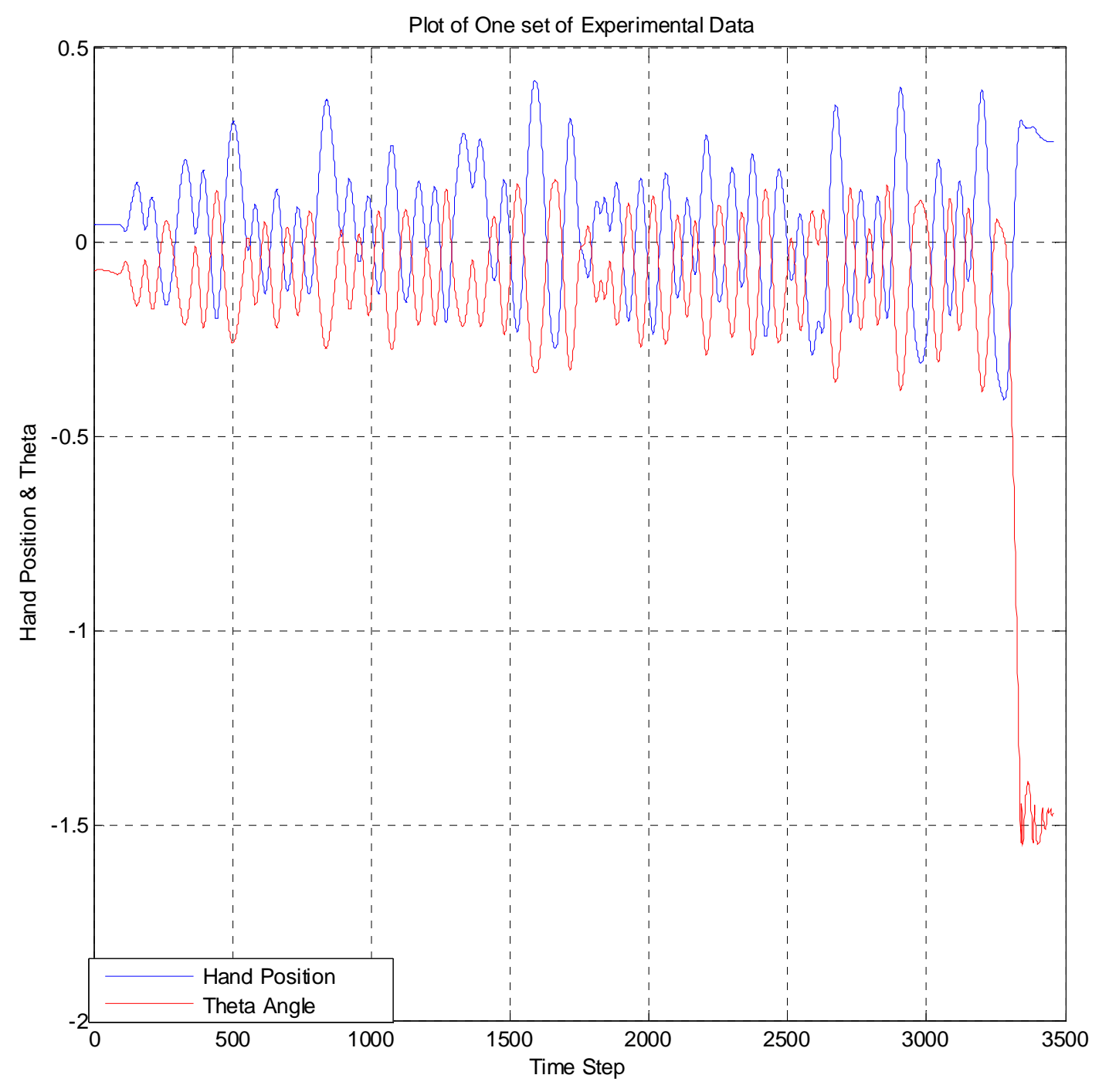

Figure 7 Plot of One Set of Experimental Data 


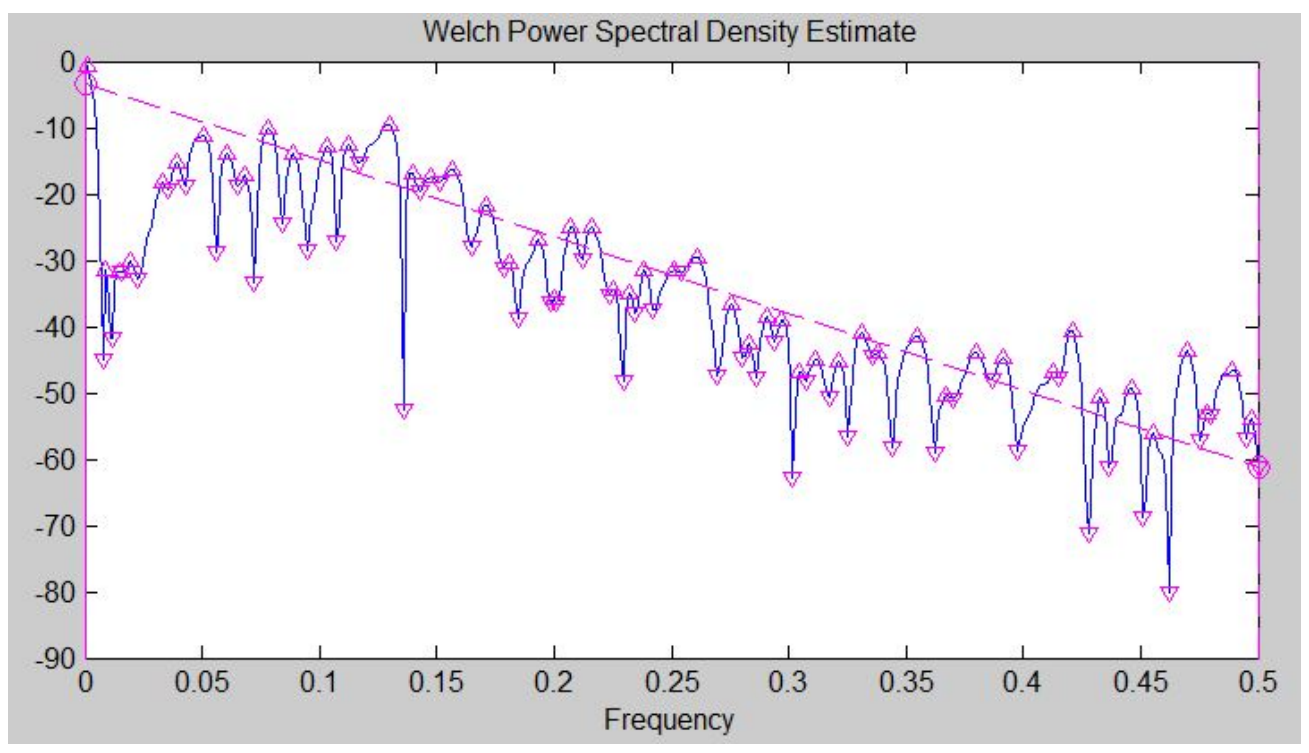

Figure 8 Power Density Spectrum of the Theta Angle

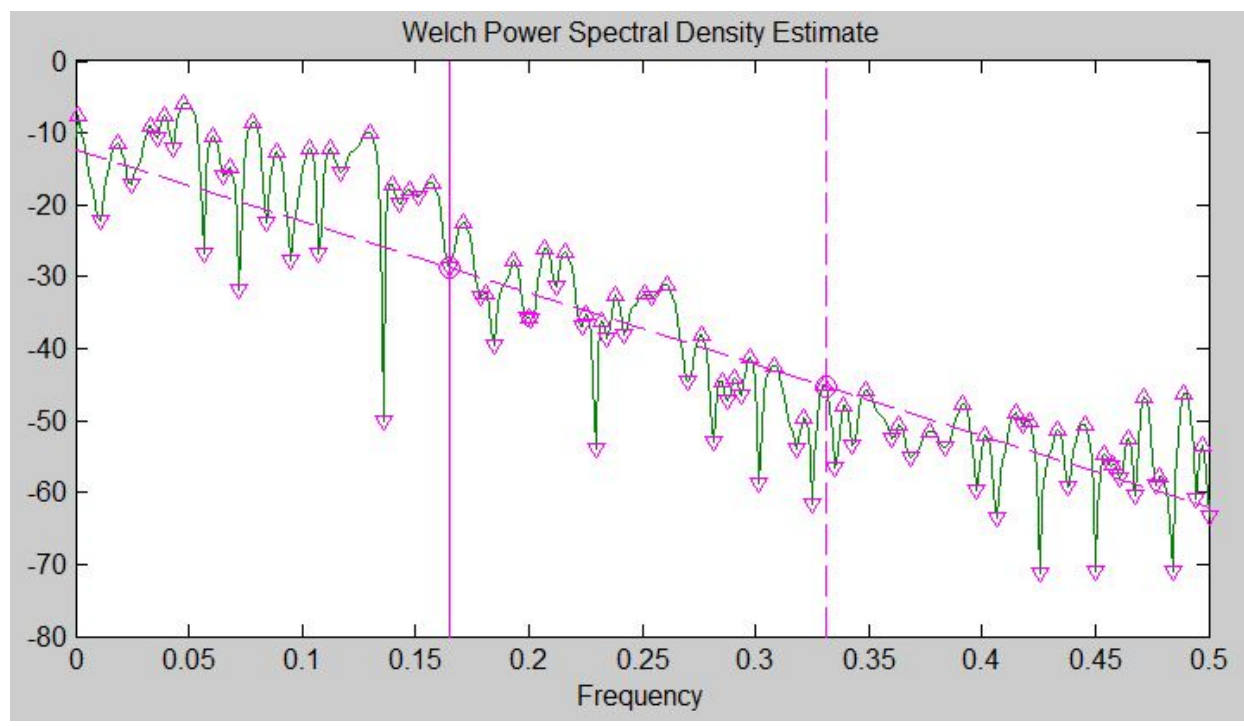

Figure 9 Power Density Spectrum of the Subject's Hand Movement 


\section{CHAPTER 4 MODELING OF THE HUMAN-INVERTED PENDULUM SYSTEM}

Recall from chapter 1 that the second objective of this study is to model the human-inverted pendulumcart system when human subjects are balancing the inverted pendulum. A good simulation is an accurate representation of a physical system. To build a good simulation model of any physical system, acquiring a good mathematical representation of such a system is imperative. In this chapter, the mathematical model for the inverted pendulum was first derived by applying the LaGrange's method to the inverted pendulum cart system. Then a mathematical model for the human operator was found from literature review. Abundant literature in the neuroscience, pilot modeling and human factors have tackled the challenge of coming up with a good mathematical model for human operators when they are undertaking different control tasks.

\subsection{Modeling of the Inverted Pendulum}

The schematic of the inverted pendulum-cart system is shown in figure 10 . The cart can only slide on a single axis rail. The control force $u$ is applied to the cart by the human subject's hand. Assume that the center of gravity of the inverted pendulum is at its geometric center. Applying LaGrange's method to the system, the Equations of Motion are found to be equation (1) \& (2). A detailed derivation of these two equations using the LaGrange's method can be found in appendix one. Table 1 is a summary of the physical quantities involved in the inverted pendulum setup and their respective values.

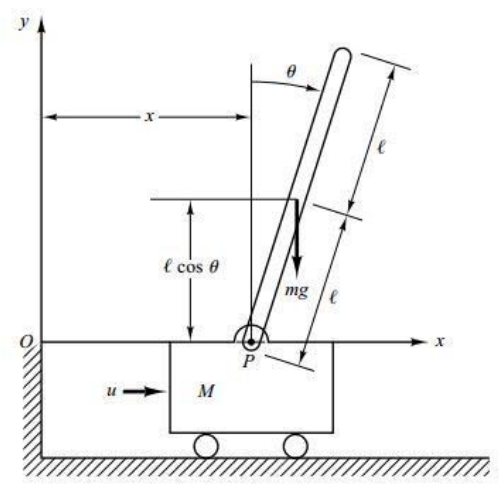

Figure 10 Schematic of the Inverted Pendulum-Cart System 


\begin{tabular}{|c|c|c|}
\hline Quantity & Symbol & value \\
\hline Mass of the rod & $\mathrm{m}$ & $0.108 \mathrm{Kg}$ \\
\hline Mass of the slide & $\mathrm{M}$ & $0.326 \mathrm{Kg}$ \\
\hline $\begin{array}{c}\text { Length of the inverted } \\
\text { pendulum }\end{array}$ & $2 \mathrm{l}$ & Variable \\
\hline $\begin{array}{c}\text { Human subject input } \\
\text { Angle displacement from the } \\
\text { vertical }\end{array}$ & $\mathrm{u}$ & Variable \\
\hline $\begin{array}{c}\text { Distance from the center of } \\
\text { the rail }\end{array}$ & $\mathrm{x}$ & $1.168 \mathrm{~meters}$ \\
\hline $\begin{array}{c}\text { Length of the rail } \\
\text { Earth's sea-level gravitational } \\
\text { constant }\end{array}$ & $\mathrm{g}$ & $9.8 \mathrm{~m} / \mathrm{s}^{2}$ \\
\hline
\end{tabular}

Table 2 Physical Characteristics of the Inverted Pendulum

$$
\begin{gathered}
m \ddot{x}+M \ddot{x}+m l \ddot{\theta}=u(\mathrm{t}) \\
m l \ddot{x}+\left(m l^{2}+I_{m}\right) \ddot{\theta}-m g l \theta=0
\end{gathered}
$$

After taking the Laplace transform of equations (1) and (2) and assuming zero initial conditions, we get

$$
\begin{gathered}
(m+M) s^{2} x(s)+m l^{2} \theta(s)=u(s) \\
m l s^{2} x(s)+\left(m l^{2}+I_{m}\right) s^{2} \theta(s)-m g l \theta(s)=0
\end{gathered}
$$

Rearrange equations (3) and (4), we get the following new transfer functions

$$
\frac{\theta(s)}{u(s)}=\frac{m l}{(m+M) m g l-\frac{1}{3} m(m+4 M) l^{2} s^{2}}
$$




$$
\frac{x(s)}{u(s)}=\frac{m g l-\left(m l^{2}+I_{m}\right) s^{2}}{(m+M) m g l s^{2}-\frac{1}{3} m(m+4 M) l^{2} s^{4}}
$$

Where $I_{m=\frac{1}{3} m l^{2}}$, thus the above two transfer functions can be further simplified into

$$
\begin{aligned}
& \frac{\theta(s)}{u(s)}=\frac{1}{(m+M) g-\frac{1}{3}(m+4 M) l s^{2}} \\
& \frac{x(s)}{u(s)}=\frac{3 g-4 l s^{2}}{3(m+M) g s^{2}-(m+4 M) l s^{4}}
\end{aligned}
$$

These two transfer function imply that the human-inverted pendulum is a single input and multipleoutput system. This means that balancing the inverted pendulum is a two-fold task, namely keeping the pendulum near the vertical place and maintaining the horizontal position of the cart within the range of the rail. For practical purposes, in this study, only the relationship between the human input and the pendulum angular output will be considered, i.e. only equation (7) will be investigated.

\subsection{Modeling of the Human Controller}

Obtaining a good mathematical model of the human operator is of critical importance in building a closed-loop human-inverted pendulum Matlab simulation and for the analysis of the stability of the system. In the section above, a mathematical model of the inverted pendulum was derived. The problem then becomes how to come up with an accurate model of the human subject. The adaptability of the human operator makes manual control very flexible. The mathematical model of the human controller thus depends heavily on the control task. In the task of balancing the inverted pendulum, the human operator can be divided into three modules which can be modeled individually. The three modules that characterize the human subject are the human visual perception system, the central nervous system and the neuromuscular system. The visual perception system is very critical in the task 
of human subjects balancing the inverted pendulum. A mathematical model of how humans estimate the motion of moving objects is thus the first step toward modeling the human operator. Humans and primates utilize three basic kinds of movements to accomplish the goal of following the movements of unpredictable targets. These eye movements are saccadic movements (step-like), pursuit movements (ramp-like), or combinations of the two[26]. Smooth pursuit eye movements allow primates to track slow moving objects. Two important characteristics of human visual perception system are that, first it is time-delayed; secondly, it is not perfect, i.e. noisy. Many have tried to quantify the amount of time delay and study the effects of such delay in reaching movements planning. Lisberger \& Westbrook found that the onset of smooth pursuit eye movements evoked by smooth target motion has latencies of 80 to 130 $m s$, depending on a number of visual parameters, including the target's luminance, size, and initial position in the visual field[27]. More recently, John et al. from UCLA investigated the progression of the visual signal in the brain while gaze correctly pursues the target (see figure 11) and the effects of this delay[28]. A model of how humans sense the velocity of moving images is proposed in this paper[29]. The authors constructed a system for processing dynamic visual inputs, which assigns velocities to components of the inputs.

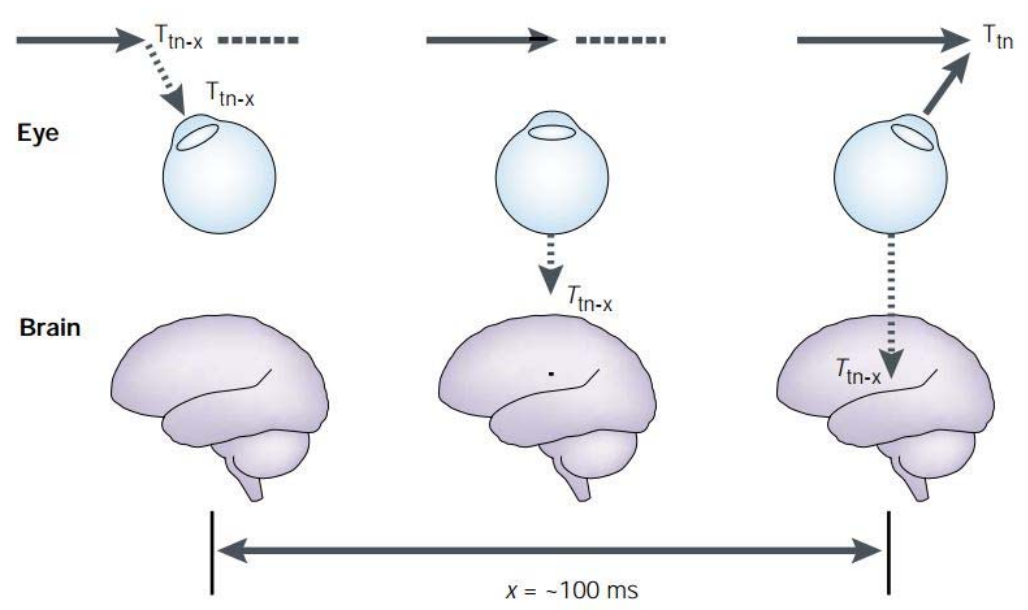

Figure 11 Schematic of the Progress of the Visual Signal in the Brain [28] 
The second essential module which distinguishes the human operator from an industrial automatic controller is the human central nervous system (CNS). The CNS is a highly optimal and computationally efficient system. It takes in the sensory information from the visual system and sends out motor command to the hand. There are different, although not competing, theories concerning how the CNS utilizes the sensory information and how it issues motor commands. The feedback mechanism is a wellestablished and supported one among them. Some other important ones include feedforward and internal model. For issuing motor commands, there are theories supporting either a continuous or an intermittent model. There seems to be some consensus that whichever model may be fitting is context dependent. Also, all these CNS computation models are highly optimal and efficient. Adult human subjects with normal motor skills demonstrated very little difficulty in the task, albeit mastering such task might require a substantial amount of training. However, this is beyond the scope of this project.

Computational models for motor control fall under the general classification of two theories: Internal model and intermittent feedback control. "Internal models are neural mechanisms that can mimic the input/output characteristics, or their inverses, of the motor apparatus[30]." "There are two varieties of the internal model: forward models, which mimic the causal flow of a process by predicting its next state given the current state and the motor command; and inverse models, which invert the causal flow by estimating the motor command that caused a particular state transition.[31]" The third module that's involved is the human neuromuscular system, which enables the execution of hand movements. As the end effector, it receives motor command from the CNS, like the visual system, time delay is also a defining characteristic of the motor system. The axons of motor neurons stretch from the brain and spinal cord to muscles or glands of the body.

To find a mathematical model for the human operator from first principles is a very hard problem considering the complexities of the human visual perception system, the CNS and the neuromuscular 
system. A mathematical model of the human operator from the engineering perspective can be more readily obtained by examining the input to and the output from the human operator. Because the human operator is able to adjust their characteristics to meet system requirements, this makes manual control system very flexible and intelligent. But the adaptability and nonlinearity of the human operator also make it difficult to describe and model mathematically the characteristics of manual control[32]. The primary reason for including humans in complex control loops is to utilize the adaptability and optimality of the human operator. This paper reviewed the rapid adaptability of human control in handling four different kinds of adaptive situations, namely input adaptation, controlled element adaptation, task adaptation and programed adaptation. "Input adaptation refers to man's ability to detect familiar or repeated patterns in the input and track these in a predictive or open loop fashion. Controlled element adaptation, as its name suggests, is human operator's ability to adapt different control strategies appropriate to changing dynamics of the system being controlled. Task adaptation is the optimization of the manual control loop on the basis of various control objectives. Finally, programmed adaptation, is used by human to adopt control strategies which he has been taught as appropriate for the situation"[33]. "A major characteristic of the input to which human adapts is the bandwidth of the input signal. Human operators respond differently in tracking low frequency inputs than they do with more difficult high frequency inputs. Specifically, the gain term is notably reduced as the input frequency cut-off increased, and the amount of lag is also reduced"[32].

McRuer et al. did much of the pioneering study in pilot modeling and quantitatively studied the effect of input bandwidth on the quasi-linear describing function model of the human operator. The general describing function for the human operator is called the crossover model, which can be used for a variety of controlled elements and forcing functions. Mathematically the describing function is expressed by the following equation[34]: 


$$
Y_{p}(j \omega) Y_{c}(j \omega)=\frac{\omega_{c} e^{-j \omega \tau_{e}}}{j \omega} \tau_{e}
$$

"The operator adjusts his or her gains and lead-lag compensation to produce a pilot describing function $Y_{c}(j \omega)$ such that, when cascaded with the controlled element $Y_{c}(j \omega)$, the open loop describing function has the characteristics of a delay and an integrator in the region of open loop gain equal to unity. Both the crossover frequency $\omega_{c}$ and the effective time delay $\tau_{e}$ show the effect of input adaptation to varying forcing function bandwidth. To track higher frequency inputs, the crossover frequency increases, as long as such increase results in lower errors. Limits of maximum crossover frequency are set by system stability condition. Reduction in the effective time delay with increasing input bandwidth is also an important aspect of input adaptation in the McRuer's crossover model" [35].

The crossover model with respect to the specific controlled element in this study, i.e. the inverted pendulum is given by the following so-called lead-lag model, which is also developed by McRuer[34].

$$
Y_{p}=K \frac{\left(\mathrm{T}_{1} \mathrm{j} \omega+1\right)}{\left(\mathrm{T}_{2} \mathrm{j} \omega+1\right)} e^{-j \omega \tau_{e}}
$$

Where $K_{p}$ is the human operator gain, $T_{1}$ the lead coefficient, $T_{2}$ the lag coefficient, $\tau_{e}$ the effective delay. This is the mathematical model that will be used to represent the human operator's dynamics in this study. By now, both the models for the inverted pendulum and the human subject have been obtained. These two mathematical models together form the mathematical model for the closed-loop human-inverted pendulum system. In next chapter, the stability of this closed-loop system will be analyzed. 


\section{CHAPTER 5 STABILITY ANALYSIS OF THE HUMAN-INVERTED PENDULUM SYSTEM}

Perhaps the most important characteristic concerning a control system is the stability condition of such a system. That is, under what conditions will a system become unstable? If it is unstable, how should we stabilize the system? Stability is the system's ability to keep close to the original trajectories when the dynamical system is under small perturbations. This stability of a system can be defined with respect to a given equilibrium point in the state space. This is also referred to as the internal stability of a system. Exterior stability or BIBO (bounded-input, bounded-output) stability also involves external input. A system is BIBO stable if for zero initial conditions, a bounded input always evokes a bounded output. BIBO stability is not just an observation that a certain bounded input generates bounded output. All bounded inputs must generate bounded outputs in order to prove that the system has BIBO stability. For linear time-invariant (LTI) systems, there are many theories and stability criteria one can employ to examine the stability of such systems, such as the Nyquist stability criterion and the Routh-Hurwitz stability criterion. It may also be determined by means of root locus analysis. Relative stability condition can be gained from examining the gain margin and phase margin from the Bode plots of the system.

For a LTI system, it is stable if and only if all closed-loop poles lie in the left-half s plane. The transfer functions of most closed-loop systems assume the following form

$$
\frac{C(\mathrm{~s})}{R(\mathrm{~s})}=\frac{b_{0} s^{m}+b_{1} s^{m-1}+\cdots+b_{m-1} s+b_{m}}{a_{0} s^{n}+a_{1} s^{n-1}+\cdots+a_{n-1} s+a_{n}}
$$

Where the a's and b's are constants and $m \leq n$.

Routh-Hurwitz Stability Criterion enables us to determine the number of closed-loop poles that lie in the right-half s plane without having to factor the denominator polynomial. The stability criterion applies to polynomials with only a finite number of terms. Information about absolute stability can be obtained 
directly from the coefficients of the characteristics equation. The necessary but not sufficient condition that all the roots have negative real parts is that all the polynomial coefficients must be nonzero and have the same sign, either negative or positive. If this condition is satisfied, then compute the RouthHurwitz array. The necessary condition that all roots of the polynomial have negative real parts is that all the elements of the first column of the array have the same sign. The number of changes of sign equals the number of roots with positive real parts [41].

\subsection{Stability Analysis of the Closed-loop Human-Inverted Pendulum System}

Figure 12 is the block diagram of the human-inverted pendulum closed-loop system. Unfortunately, the system operates in the presence of delay, primary due to the time the human operator takes to acquire the visual information needed for issue the motor command. It is apparent to see from chapter 2 that the human operator is inherently time-delayed. There are systematic and measurable delays in the human visual perception system, the central nervous system and the neuromuscular system. The stability study of systems with time delay has been a very active and fruitful endeavor. Many tests and criteria have been established to investigate the stability of time-delay systems.

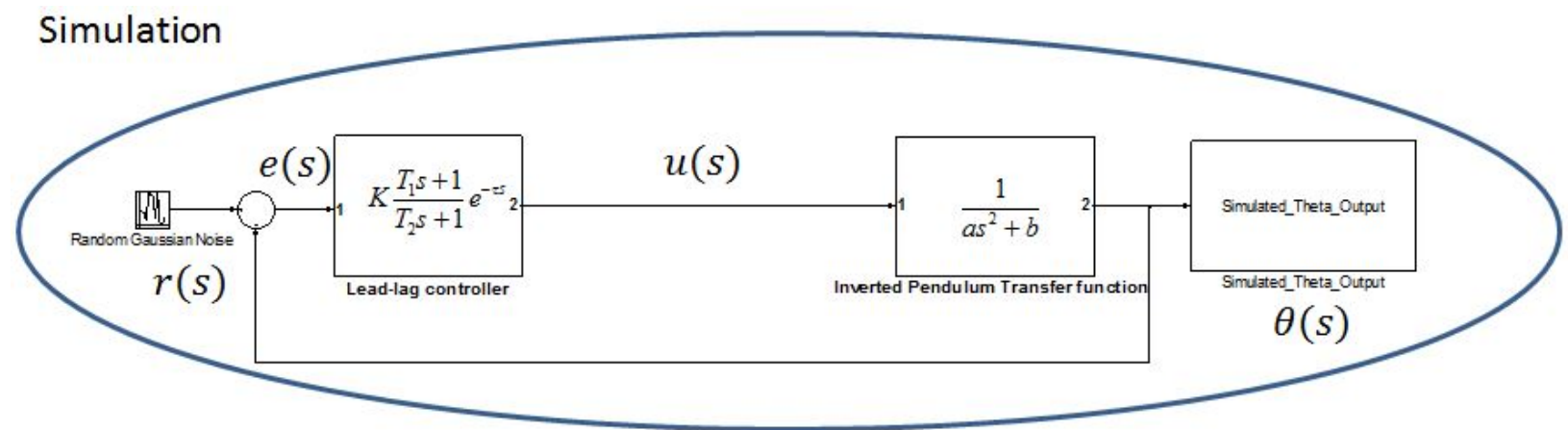

Figure 12 Block diagram of the Closed-loop Human-inverted Pendulum

The time delayed mathematical model assumed for the human subject in this study is the lead-lag model given by equation (12). 


$$
\frac{u(s)}{e(s)}=K \frac{T_{1} s+1}{T_{2} s+1} e^{-\tau s}
$$

The transfer function for the inverted pendulum is given by the following equation.

$$
\frac{\theta(s)}{u(s)}=\frac{1}{(m+M) g-\frac{1}{3}(m+4 M) l s^{2}}
$$

Let's denote

$$
a=-\frac{1}{3}(m+4 M) l=-0.2696 \text { and } b=(m+M) g=4.267
$$

The closed loop then becomes

$$
\frac{\theta(s)}{r(s)}=\frac{K\left(T_{1} s+1\right) e^{-\tau s}}{\left(a s^{2}+b\right)\left(T_{2} s+1\right)+K\left(T_{1} s+1\right) e^{-\tau s}}
$$

The polynomial of the denominator is not rational because of the transcendental exponential term. This makes determining the ranges of K, T1 and T2 more difficult. In order to apply Routh-Hurwitz stability criterion to the polynomial in the denominator, we need first transfer it into an algebraic function, which only has rational coefficients. This can be done by applying a first order pade approximation to the exponential term. A pade approximant is the best approximation of a function by a rational function of given order. The pade approximant often gives better approximation of the function than truncating its Taylor series. The form for the first order pade approximation is given by the following equation.

$$
e^{-\tau s} \approx \frac{1-\frac{\tau s}{2}}{1+\frac{\tau s}{2}}
$$

Let's denote $\frac{\tau S}{2}=\tau_{1} s$ and substitute equation (16) into equation (15), the transfer function then becomes 


$$
\frac{\theta(s)}{r(s)}=\frac{K\left(T_{1} s+1\right)\left(1-\tau_{1} s\right)}{\left(a s^{2}+b\right)\left(T_{2} s+1\right)\left(1+\tau_{1} s\right)+K\left(T_{1} s+1\right)\left(1-\tau_{1} s\right)}
$$

Expand the denominator, we get.

$$
\frac{\theta(s)}{r(s)}=\frac{K\left(T_{1} s+1\right)\left(1-\tau_{1} s\right)}{a T_{2} \tau_{1} s^{4}+a\left(T_{2}+\tau_{1}\right) s^{3}+\left(a+b T_{2} \tau_{1}-K T_{1} \tau_{1}\right) s^{2}+\left(b T_{2}+b \tau_{1}+K T_{1}-K \tau_{1}\right) s+b+K}
$$

The Routh array of the polynomial in the denominator is

$$
\begin{array}{ll}
s^{4} & a T_{2} \tau_{1} \quad\left(a+b T_{2} \tau_{1}-K T_{1} \tau_{1}\right) \quad(\mathrm{b}+K) \\
s^{3} & a\left(T_{2}+\tau_{1}\right) \quad\left(b T_{2}+b \tau_{1}+K T_{1}-K \tau_{1}\right) \\
s^{2} & \frac{a T_{2}+a \tau_{1}-2 K T_{1} T_{2} \tau_{1}-K T_{1} \tau_{1}^{2}+K T_{2} \tau_{1}^{2}}{\left(T_{2}+\tau_{1}\right)} \quad(\mathrm{b}+K) \\
s^{1} & \frac{\left(a T_{2}+a \tau_{1}-2 K T_{1} T_{2} \tau_{1}-K T_{1} \tau_{1}^{2}+K T_{2} \tau_{1}^{2}\right)\left(b T_{2}+b \tau_{1}+K T_{1}-K \tau_{1}\right)-a\left(T_{2}+\tau_{1}\right)^{2}(\mathrm{~b}+K)}{a T_{2}+a \tau_{1}-2 K T_{1} T_{2} \tau_{1}-K T_{1} \tau_{1}^{2}+K T_{2} \tau_{1}^{2}} \\
s^{0} & (\mathrm{~b}+K)
\end{array}
$$

Apply the Routh stability criterion to the coefficients of the polynomial in the denominator and the first column of the Routh array we get the following seven inequalities. 


$$
\begin{aligned}
& a T_{2} \tau_{1}>0,(1) \\
& \left(a+b T_{2} \tau_{1}-K T_{1} \tau_{1}\right)>0,(2) \\
& a\left(T_{2}+\tau_{1}\right)>0,(3) \\
& \left(\mathrm{b} T_{2}+b \tau_{1}+K T_{1}-K \tau_{1}\right)>0,(4) \\
& (\mathrm{b}+K)>0,(5) \\
& \frac{a T_{2}+a \tau_{1}-2 K T_{1} T_{2} \tau_{1}-K T_{1} \tau_{1}^{2}+K T_{2} \tau_{1}^{2}}{\left(T_{2}+\tau_{1}\right)}>0,(6) \\
& \frac{\left(a T_{2}+a \tau_{1}-2 K T_{1} T_{2} \tau_{1}-K T_{1} \tau_{1}^{2}+K T_{2} \tau_{1}^{2}\right)\left(b T_{2}+b \tau_{1}+K T_{1}-K \tau_{1}\right)-a\left(T_{2}+\tau_{1}\right)^{2}(\mathrm{~b}+K)}{a T_{2}+a \tau_{1}-2 K T_{1} T_{2} \tau_{1}-K T_{1} \tau_{1}^{2}+K T_{2} \tau_{1}^{2}}>0,(7)
\end{aligned}
$$

This is a set of multivariate polynomial inequalities. It is very hard to solve this set of multivariate polynomial inequalities, in order to obtain the ranges of $\mathrm{K}, \mathrm{T} 1$ and $\mathrm{T} 2$. However, we can check the necessary condition for the system to be stable first. For this, we only have to solve the inequalities (1) to (5). Solving the first five inequalities gives us the following conditions of $K, T 1$ and $T 2$.

$$
\begin{aligned}
& T_{2}<-\tau_{1} \\
& K>-b \\
& \frac{K-b}{K} \tau_{1}>\frac{a}{K \tau_{1}} \\
& \frac{(K-b) \tau_{1}^{2}-a}{2 b \tau_{1}}<-\tau_{1}
\end{aligned}
$$

This upon further simplification becomes

$$
\begin{aligned}
& T_{2}<-\tau_{1},(1) \\
& K<\frac{a}{\tau_{1}^{2}}+b,(2) \\
& K<\frac{a}{\tau_{1}^{2}}-b,(3) \\
& K>-b,(4)
\end{aligned}
$$


Notice, however, inequality (3) and (4) contradicts with each other because $\frac{a}{\tau_{1}^{2}}-b$ is always less than $-b$, since $a$ is always less than zero. Thus inequality (3) and (4) cannot both be satisfied. This yields no solution for $\mathrm{K}$, thus no solution for $\mathrm{T} 1, \mathrm{~T} 2$ either.

On the surface, it seems that it's impossible to obtain absolute stability for the closed-loop system when the time delay is added. However, assuming the coefficients of the polynomial in the denominator are all negative, we have the following five inequalities.

$$
\begin{aligned}
& a T_{2} \tau_{1}<0 \\
& \left(a+b T_{2} \tau_{1}-K T_{1} \tau_{1}\right)<0 \\
& a\left(T_{2}+\tau_{1}\right)<0 \\
& \left(\mathrm{~b} T_{2}+b \tau_{1}+K T_{1}-K \tau_{1}\right)<0 \\
& (\mathrm{~b}+K)<0
\end{aligned}
$$

Solving these five inequalities, we get the following conditions for K, T1 and T2.

$$
\begin{aligned}
& K<-b \\
& T_{2}>-\tau_{1} \cap T_{2}>0 \\
& \frac{K-b}{K} \tau_{1}<T_{1}<\frac{a}{K \tau_{1}} \\
& \frac{(K-b) \tau_{1}^{2}-a}{2 b \tau_{1}}>T_{2}
\end{aligned}
$$

Upon further simplification, they become.

$$
\begin{aligned}
& \frac{a}{\tau_{1}^{2}}+b<K<-b \\
& 0<T_{2}<\frac{(K-b) \tau_{1}^{2}-a}{2 b \tau_{1}} \\
& \frac{K-b}{K} \tau_{1}<T_{1}<\frac{a}{K \tau_{1}}
\end{aligned}
$$


Let's check again to see if they yield solutions for K, T1 and T2. In order to obtain a range for K, we need

$$
\frac{\mathrm{a}}{\tau_{1}^{2}}+\mathrm{b}<-\mathrm{b}
$$

This yields

$$
\tau_{1}^{2}<\frac{-2 \mathrm{a}}{\mathrm{b}}
$$

Or $\tau_{1}<0.177$, after we substitute $a=-0.2696$ and $b=4.267$ into the above equation.

Equation (27) gives us a few interesting insights as how the human controller adapt to the control task. Notice, $\tau_{1}$ is solely determined by $a$ and $b$, which are the characteristics of the inverted pendulum. Equation (27) shows that for a given inverted pendulum setup, there is a maximum delay that is allowed in the human controller if the mathematical model for the human controller is a lead-lag plus delay model. Once the delay in the human controller exceeds the maximum delay allowed for whatever reasons, e.g. fatigue, loss of attention etc., the human subject won't be able to stabilize the pendulum no matter how he or she adjust the other parameters in the model, namely gain $\mathrm{K}$, lead T1 and lag T2. The maximum allowable delay in the inverted pendulum setup in this study is about $0.354 \mathrm{~s}$. The amount of delay in the human controller is hard to quantify when the subject is balancing the pendulum. For practical reason, it's hard to check the ranges of T1 and T2, when both the time delay $\tau$ and gain $K$ are varied. Three different and representative values for the time delay are chosen and then investigated to see how they affect the ranges of $\mathrm{K}, \mathrm{T} 1$ and T2. The values of $\tau$ chosen in this study are $0.16 \mathrm{~s}, 0.24 \mathrm{~s}$ and $0.32 \mathrm{~s}$ respectively. Figure 13 shows the plots of $\mathrm{K}, \mathrm{T} 1$ and $\mathrm{T} 2$ when $\tau$ assumes different values. As the plot shows when the human controller exhibits little or no delay, the permissible range of $K$ is very large. As it can be seen from the plots, when the delay increases the ranges of $K, T 1$ and $\mathrm{T} 2$ are all effectively reduced by a considerable amount. Further, a set of parameters for $\tau, K, T_{1}$ 
and $T_{2}$ are chosen from the first case, which is when $\tau=0.16 \mathrm{~s}$, where, $K=-4.5, T_{1}=0.45$ and $T_{2}=0.1$. Substitute them into the closed-loop transfer function, we get

$$
\frac{\theta(s)}{r(s)}=\frac{-0.162 s^{2}+1.665 s+4.5}{0.002157 s^{4}+0.0485 s^{3}+0.07346 s^{2}+0.8969 s+0.233}
$$

The roots of the characteristic equation are $-21.8022,-0.2167 \pm 4.3227 i,-0.2645$. They all have negative real parts. As can be seen from the bode plot and the Nyquist plot of the open loop system in figure 14 , the values assumed for the parameters in the human controller can indeed stabilize the inverted pendulum.
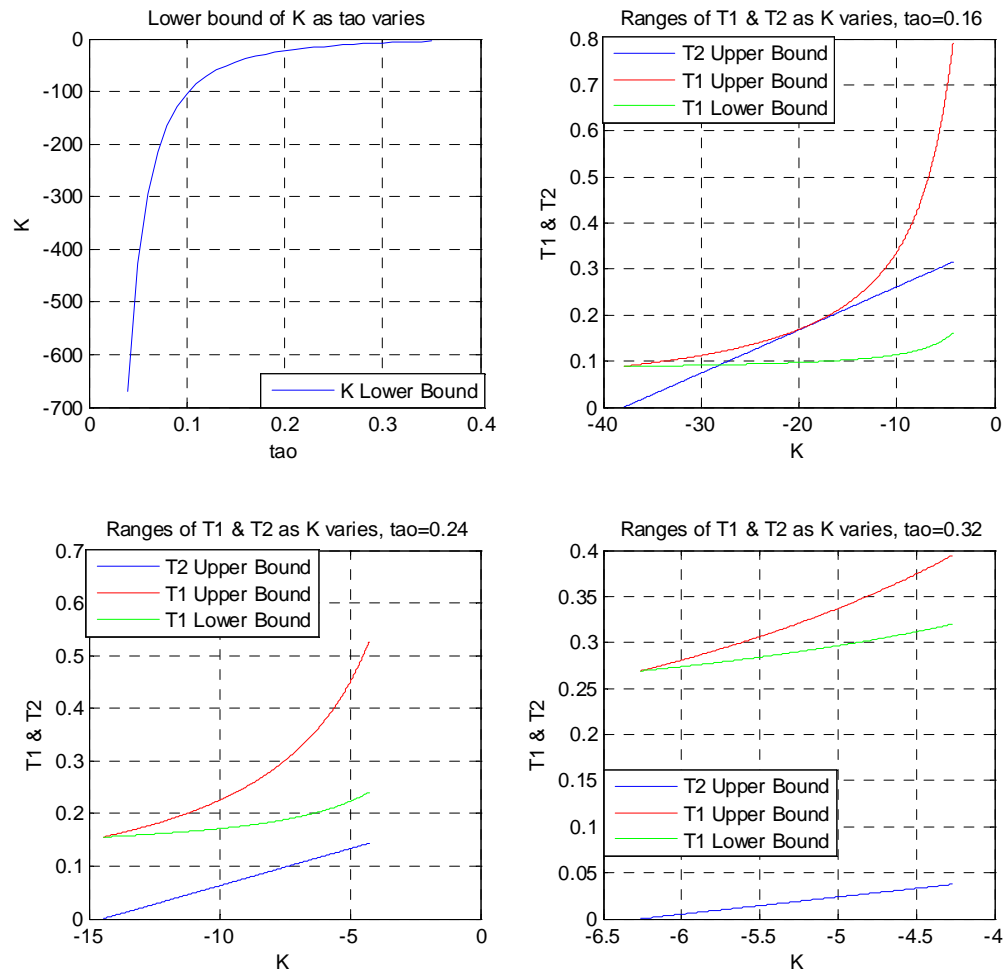

Figure 13 Stability conditions of K, T1 \& T2 with different time delays 

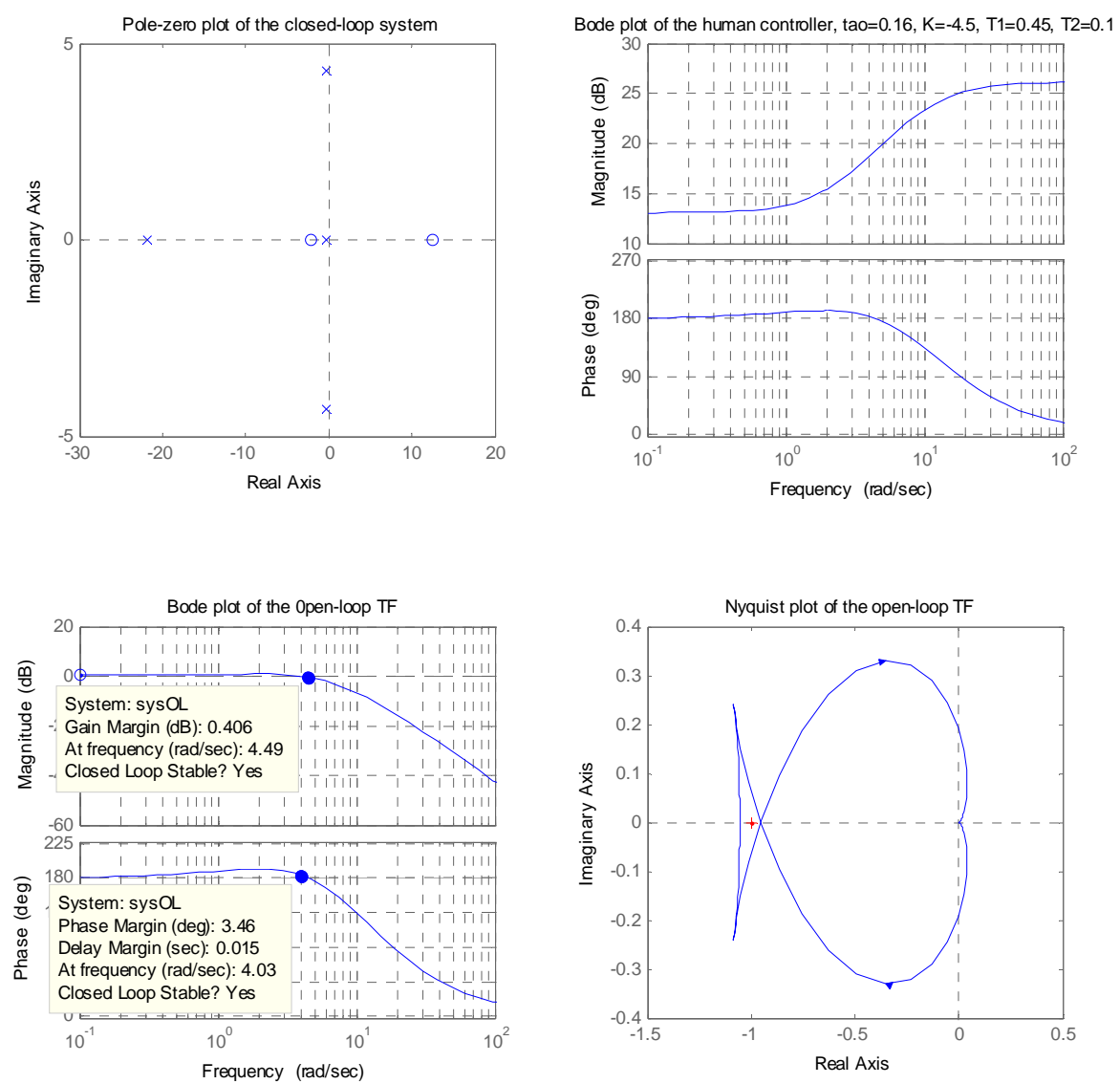

Figure 14 Plots of the stable closed-loop human inverted pendulum system

\subsection{The Simulation in Matlab \& Simulink}

Based on the stability analysis in the previous section, a simulation of the system was built in Matlab/Simulink (figure 15). A picture of the inverted pendulum animation is provided in figure 16. Later in chapter 6, the simulation will be used to verify the estimator developed and to study the stability of the system and display the results graphically. 


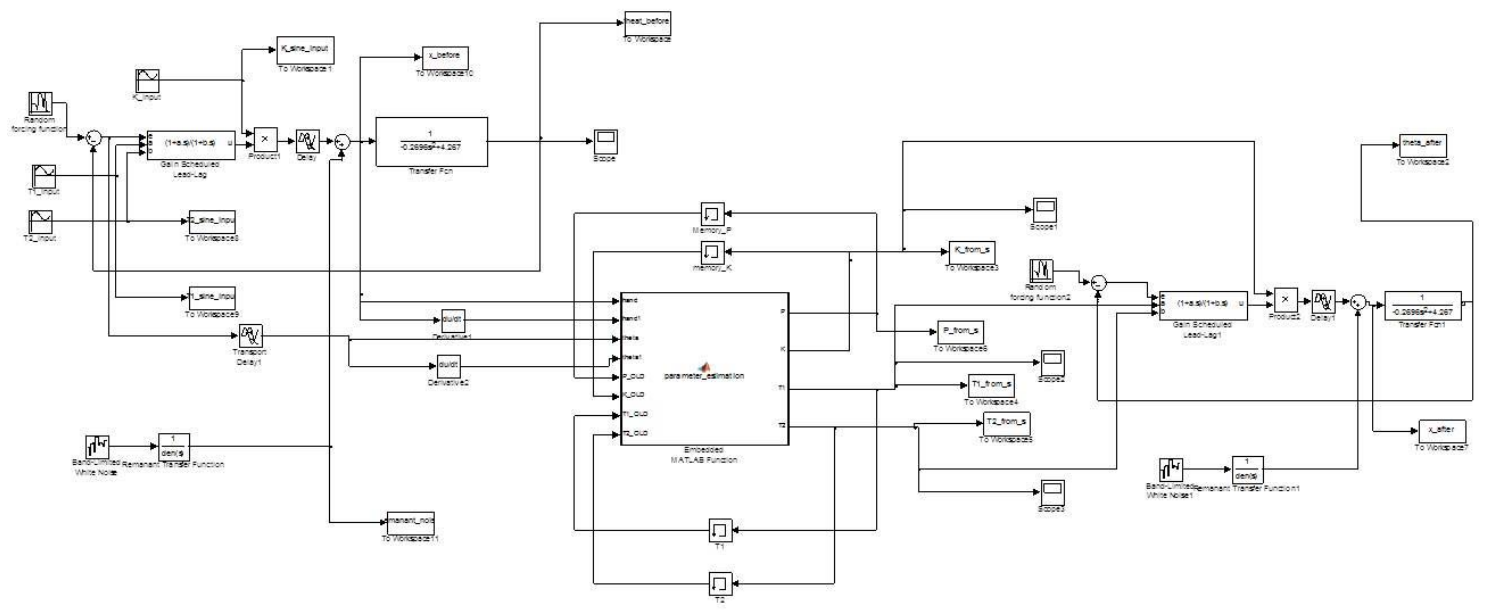

Figure 15 Matlab/Simulink Mode of the Human-Inverted Pendulum System

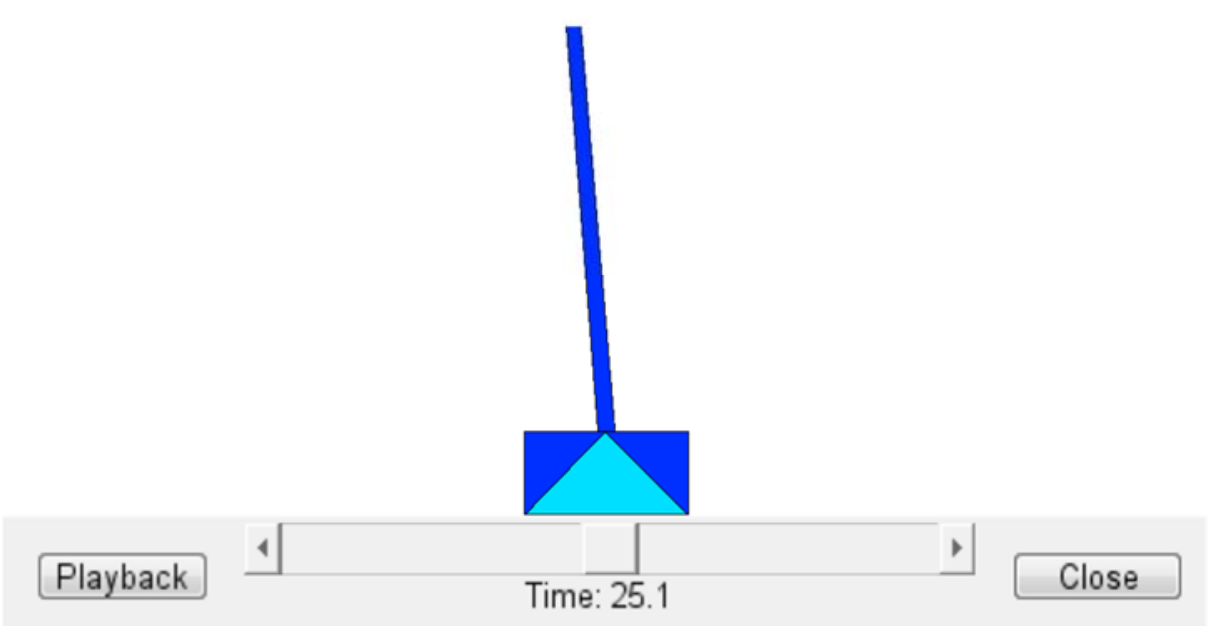

Figure 16 An animation of the Inverted Pendulum 


\section{CHAPTER 6 PARAMETERS ESTIMATION OF THE HUMAN SUBJECTS}

Estimation of the parameters in the human operator model is very valuable for the quantification of the different characteristics of manual control. The hypothesis in our study is that the changes the human subjects undergo when balancing the inverted pendulum are the reasons that the inverted pendulum eventually falls. The mathematical model assumed in this study is given by the following equation.

$$
\frac{u(s)}{e(s)}=K \frac{T_{1} s+1}{T_{2} s+1} e^{-\tau s}
$$

There are four parameters in this model. To quantify the changes the human subjects undergo thus obtain the transition of the stability of the human-inverted pendulum system, it is necessary to use estimation algorithms to estimate the values of these parameters.

\subsection{Estimation Algorithm Selection and Verification}

There are many advanced data filtering and parameters estimation algorithms available. They can be broadly divided into two categories, the time domain methods and the frequency domain methods[40]. Among the time domain estimation techniques, Extend Kalman Filter and Unscented Kalman filter are two every powerful and applicable ones. They have proven to be especially useful when the processes relating the state and the output of the system are nonlinear in nature. In this study, the Kalman filter algorithm is chosen as the estimator. The parameters of the transfer function assumed for the human subject can be estimated by applying the extended Kalman filter to the corresponding differentialdifference equations in the time domain. The Kalman filter is a set of mathematical equations, when executed recursively, provides an optimal estimate of the state of a process, in a way that minimizes the mean squared error of the state[36]. In 1960, R.E. Kalman published a seminal paper and provided a 
recursive solution to the discrete linear filtering problem[37]. Many variations of the original Kalman filter were developed for tackling problems involving nonlinearity[38, 39].

\subsubsection{The Framework of the Kalman Filter}

The Kalman filter includes two main steps, prediction and update. In the prediction step, the state and the covariance associated with the state prediction are propagated through the state transition matrix. In the update steps, the state is updated by using the innovation or residual and the posterior covariance associated with the posterior state is also calculated.

The discrete-time linear Kalman filter can be applied to a system with the following form.

$$
\begin{aligned}
\mathbf{x}_{k} & =\mathbf{A}_{k-1} \mathbf{x}_{k-1}+\mathbf{B}_{k-1} \mathbf{u}_{k-1}+\mathbf{w}_{k-1} \\
\mathbf{y}_{k} & =\mathbf{H}_{k} \mathbf{x}_{k}+\mathbf{v}_{k}
\end{aligned}
$$

Where $\mathbf{w}$ and $\mathbf{v}$ are the process and measurement noise processes, which are assumed to be white, uncorrelated, and zero-mean with known covariance matrices $\mathbf{Q}$ and $\mathbf{R}$ respectively

$$
\begin{array}{ll}
\mathbf{w}_{k} \sim\left(0, \mathbf{Q}_{k}\right), & E\left[\mathbf{w}_{k} \mathbf{w}_{j}^{T}\right]=\mathbf{Q}_{k} \delta_{k j} \\
\mathbf{v}_{k} \sim\left(0, \mathbf{R}_{k}\right), & E\left[\mathbf{v}_{k} \mathbf{v}_{j}^{T}\right]=\mathbf{R}_{k} \delta_{k j} \\
E\left[\mathbf{v}_{k} \mathbf{w}_{j}^{T}\right]=0 &
\end{array}
$$

Where $\delta$ is the Kronecker delta function

$$
\delta_{k j}= \begin{cases}1 & k=j \\ 0 & k \neq j\end{cases}
$$

The initial conditions for the filter are given by

$$
\begin{aligned}
& \hat{\mathbf{x}}_{0}=E\left[\mathbf{x}_{0}\right] \\
& \mathbf{P}_{0}=E\left[\left(\mathbf{x}_{0}-\hat{\mathbf{x}}_{0}\right)\left(\mathbf{x}_{0}-\hat{\mathbf{x}}_{0}\right)^{T}\right]
\end{aligned}
$$


The first step in executing a Kalman filter is to obtain the predictions of the current state and covariance matrix based on information from the previous step. This is called the prediction step.

$$
\begin{aligned}
& \hat{\mathbf{x}}_{k \mid k-1}=\mathbf{A}_{k-1} \hat{\mathbf{x}}_{k-1}+\mathbf{B}_{k-1} \mathbf{u}_{k-1} \\
& \mathbf{P}_{k \mid k-1}=\mathbf{A}_{k-1} \mathbf{P}_{k-1} \mathbf{A}_{k-1}^{T}+\mathbf{Q}_{k-1}
\end{aligned}
$$

Then, the Kalman gain matrix, $\mathbf{K}$, is calculated

$$
\mathbf{K}_{k}=\mathbf{P}_{k \mid k-1} \mathbf{H}_{k}^{T}\left(\mathbf{H}_{k} \mathbf{P}_{k \mid k-1} \mathbf{H}_{k}^{T}+\mathbf{R}_{k}\right)^{-1}
$$

Finally, the Kalman gain matrix is used to update the predicted state and covariance matrix using the residual from the measurement

$$
\begin{aligned}
& \hat{\mathbf{x}}_{k}=\hat{\mathbf{x}}_{k \mid k-1}+\mathbf{K}_{k}\left(\mathbf{z}_{k}-\mathbf{H}_{k} \hat{\mathbf{x}}_{k \mid k-1}\right) \\
& \mathbf{P}_{k}=\left(\mathbf{I}-\mathbf{K}_{k} \mathbf{H}_{k}\right) \mathbf{P}_{k \mid k-1}=\left(\mathbf{P}_{k \mid k-1}^{-1}+\mathbf{H}_{k}^{T} \mathbf{R}_{k}^{-1} \mathbf{H}_{k}\right)^{-1}
\end{aligned}
$$

Where $\mathbf{z}$ is the measurement of the output, $\mathbf{y}$, and $\mathbf{I}$ is an identity matrix [36].

\subsubsection{The Kalman Filter Formulation of Our Problem}

Recall from chapter 4 that the mathematical model for the human operator in our study is given by the following equation.

$$
\frac{u(s)}{e(s)}=K \frac{T_{1} s+1}{T_{2} s+1} e^{-\tau s}
$$

Where, $u(s)$ is the output of the human subject, $e(s)$ is the error input, $K$ is the time varying gain, $T_{1}$ is the time-varying lead parameter, $T_{2}$ is the time-varying lag parameter and $\tau$ is the overall time delay 
constant which can be determined from the input and output data. Take the inverse Laplace transform of the above transfer function, we get

$$
T_{2} \dot{u}(t)+u(t)=K T_{1} \dot{e}(t)+K e(t)
$$

The process model is assumed to be, because a precise of model of how $K, T_{1}$ and $T_{2}$ varies is not known.

$$
\dot{\mathbf{X}}=\left[\begin{array}{c}
\dot{K} \\
\dot{T}_{1} \\
\dot{T}_{2}
\end{array}\right]=\left[\begin{array}{l}
0 \\
0 \\
0
\end{array}\right]+\mathbf{w}(\mathrm{t})
$$

Where $\boldsymbol{w}(t)$ is a random Gaussian noise.

Our process noise covariance matrix is assumed to be

$$
\mathbf{Q}=\left[\begin{array}{ccc}
0.01 & 0 & 0 \\
0 & 0.01 & 0 \\
0 & 0 & 0.01
\end{array}\right]
$$

The state transition matrix is

$$
\mathbf{F}=\left[\begin{array}{lll}
1 & 0 & 0 \\
0 & 1 & 0 \\
0 & 0 & 1
\end{array}\right]
$$

The measurement model is given by

$$
y=u(t)=K T_{1} \dot{e}(t)+K e(t)-T_{2} \dot{u}(t)+v(t)
$$

The measurement noise variance is assumed to be.

$$
R=0.245
$$


The measurement matrix is

$$
H=\left[T_{1} \dot{\theta}(t)+\theta(t) \quad K \dot{\theta}(t)-\dot{u}(t)\right]
$$

The flow chart of executing the algorithm is given by figure 17 .

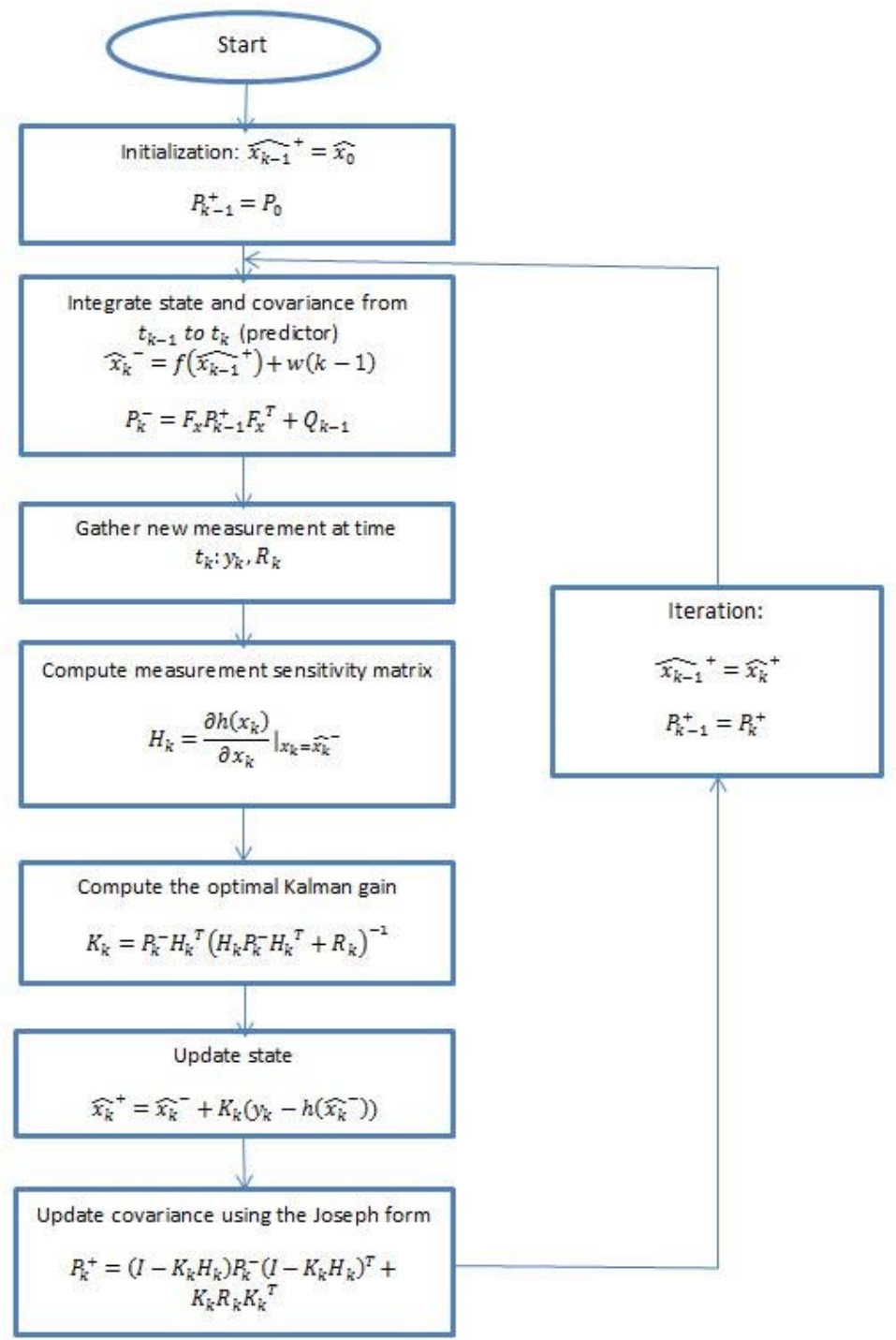

Figure 17 Flow Chart of the Extended Kalman Filter Algorithm 


\subsubsection{Filter Verification}

Before the Kalman filter developed in the section above is used to process the experimental data, it has to be verified using the simulation data. The way this is done is to preset the values of the parameters in the human controller model and then use the Kalman filter to estimate those parameters from the simulation data. If the values of the estimated parameters converge to the preset parameters, it indicates that the Kalman filter can theoretically estimate the parametric human controller model. Figure 18 provides the schematic of the Simulink simulation, which is used to generate the simulation data and to perform estimation.

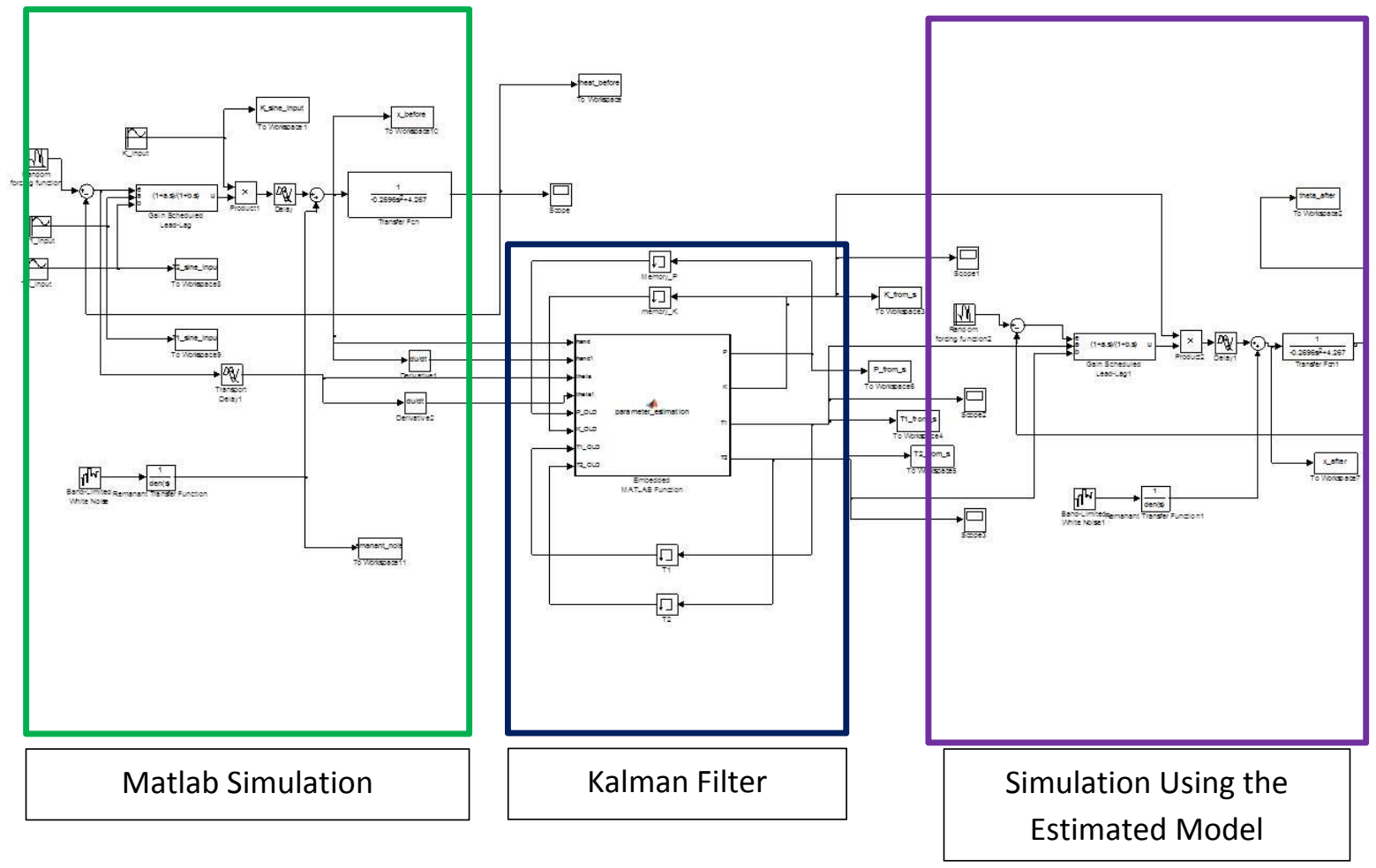

Figure 18 Matlab Scheme for Verifying the EKF 


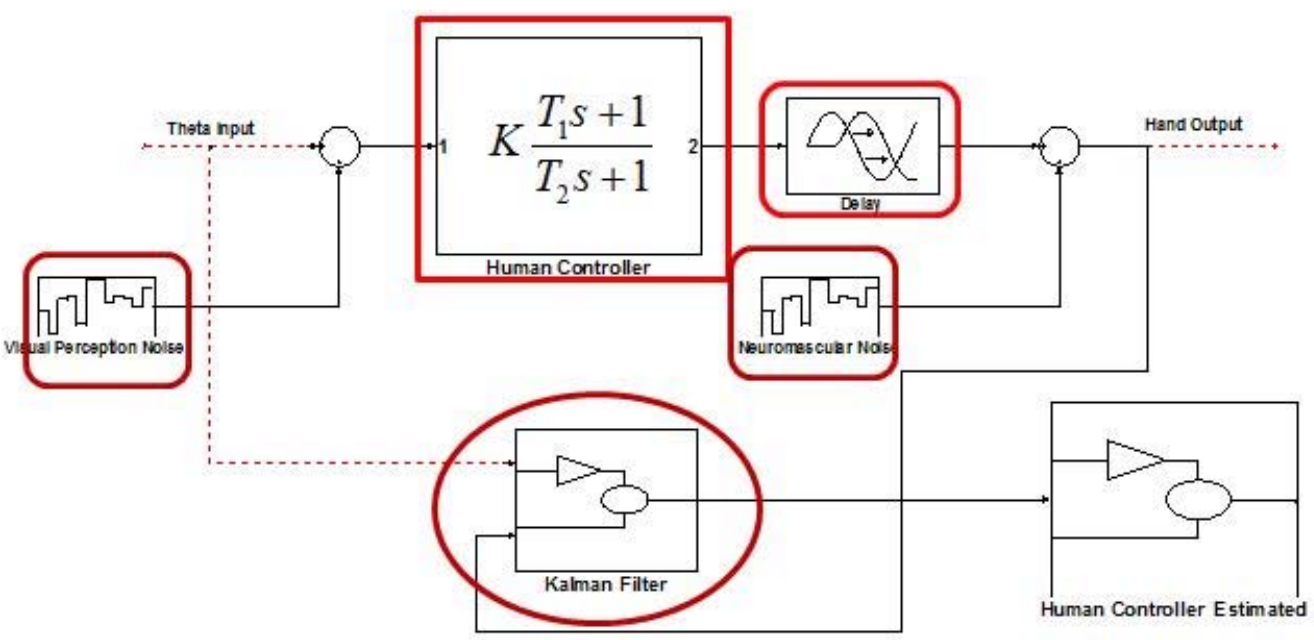

Figure 19 Block Diagram of the closed-loop system

Figure 19 is the block diagram of the human controller model. From the diagram, it can be seen that there are several factors that could affect the performance of the Kalman filter. The first factor is the noise present in the human controller. The second factor is the delay present in the model. The Third factor is the time varying nature of the model. In this section, six different cases of how individual factor affects the performance of the filter are discussed and the results from each case are presented. The values of $K, T_{1}$, and $T_{2}$ for all six cases are $-4.5,0.45$ and 0.1 respectively, which are preset in the human controller model.

\section{Case 1}

Assuming there is no delay and remnant noise in the human controller, this is the simplest scenario. The estimation results from the simulation data is plotted in figure 20 and tabulated in table 3 . From figure 20 and table 3, it can be seen that the estimation results are far away from the parameters set in the controller, no matter how the measurement variance $\mathrm{R}$ is tuned. 

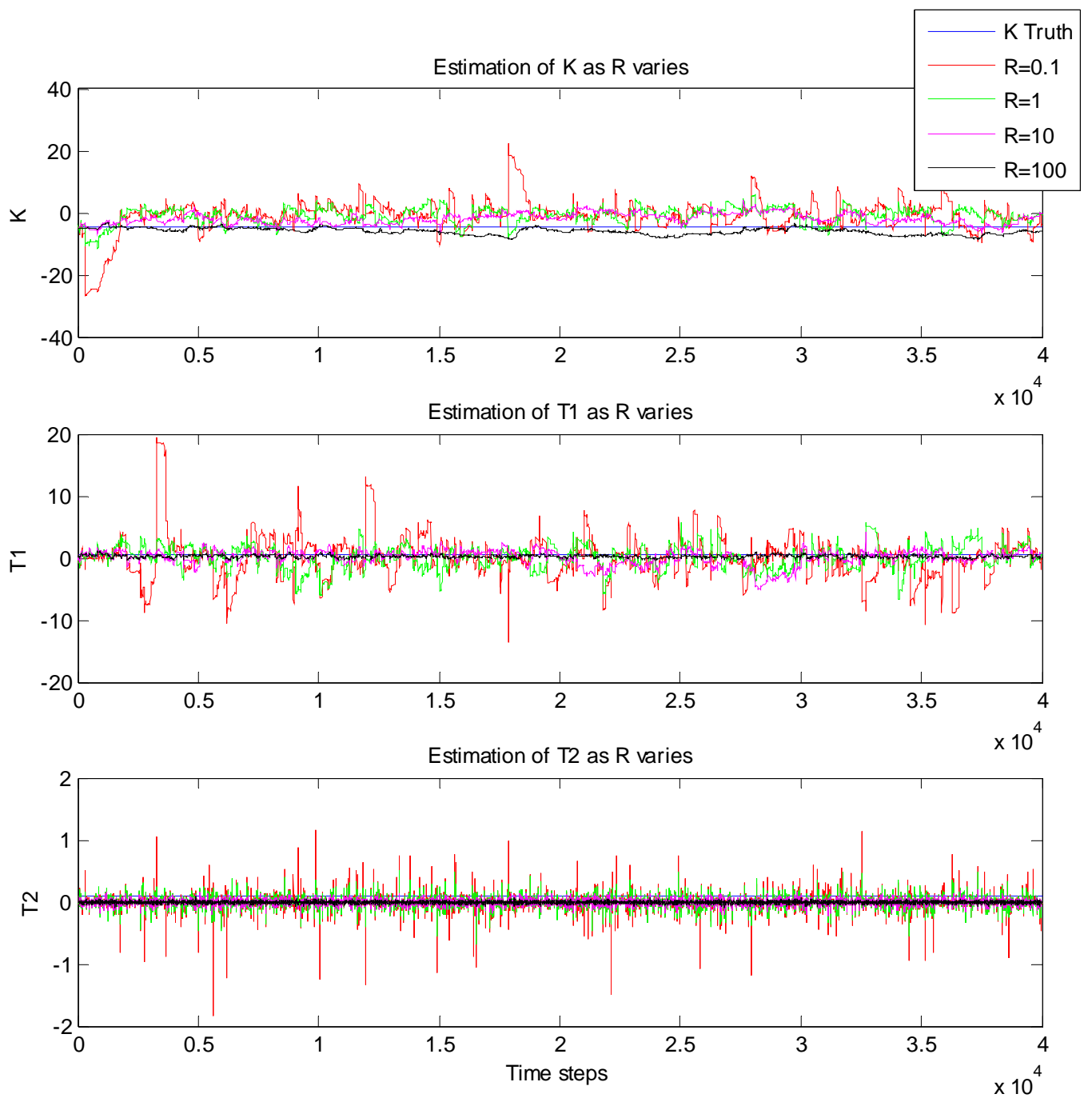

Figure 20 Plots of $\mathrm{K}, \mathrm{T} 1$ \& T2 as R varies, without noise compensated

\begin{tabular}{|c|c|c|c|c|c|}
\hline \multicolumn{2}{|c|}{} & $\mathbf{R}=0.1$ & $\mathbf{R}=1$ & $\mathrm{R}=10$ & $\mathrm{R}=100$ \\
\hline \multirow{2}{*}{$\Delta K$} & $\mu$ & 3.499 & 3.590 & 2.602 & -1.438 \\
\hline & $\sigma$ & 5.245 & 2.593 & 1.729 & 1.052 \\
\hline \multirow{2}{*}{$\Delta T 1$} & $\mu$ & -0.392 & -0.341 & -0.312 & -0.167 \\
\hline & $\sigma$ & 3.522 & 1.8965 & 1.2065 & 0.216 \\
\hline \multirow{2}{*}{$\Delta T 2$} & $\mu$ & -0.105 & -0.105 & -0.105 & -0.104 \\
\hline & $\sigma$ & 0.049 & 0.033 & 0.020 & 0.011 \\
\hline
\end{tabular}

Table 3 Mean and standard deviation of delta k, delta T1, delta T2 for case 1 


\section{Case 2}

Assuming the visual perception noise is known perfectly, thus the theta input to estimator can be compensated beforehand. As it turns out, if the visual perception noise is perfect known and compensated for the theta angle, the estimation results (figure 21) converge very quickly to the parameters set in the human controller model.
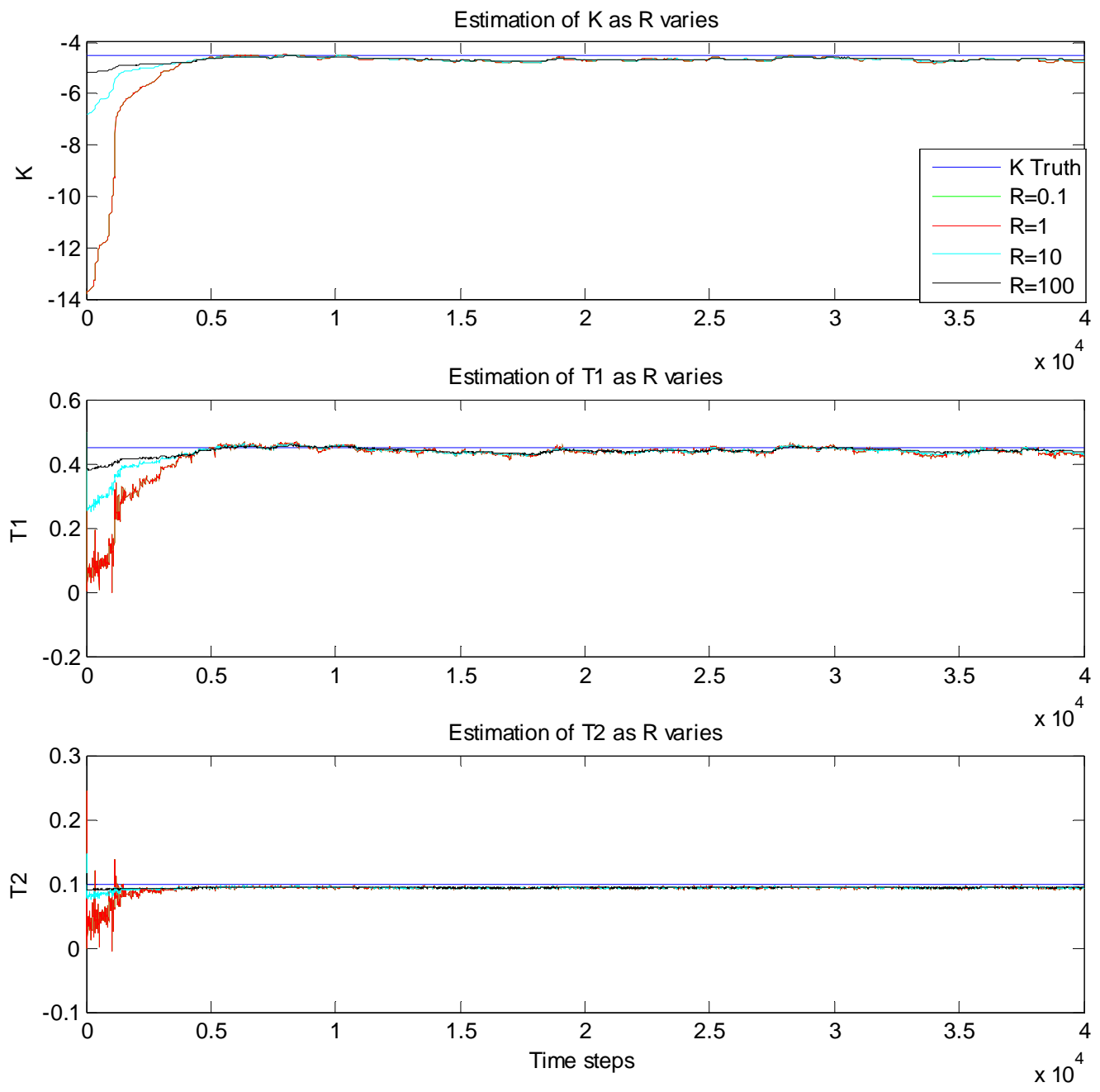

Figure 21 Estimation of $\mathrm{K}, \mathrm{T} 1$ \& T2 as R varies, with noise compensated 


\begin{tabular}{|c|c|c|c|c|c|}
\hline & $R=0.1$ & $R=1$ & $R=10$ & $R=100$ \\
\hline \multirow[t]{2}{*}{$\Delta K$} & $\mu$ & -0.166 & -0.161 & -0.154 & -0.147 \\
\hline & $\sigma$ & 0.091 & 0.075 & 0.062 & 0.050 \\
\hline \multirow[t]{2}{*}{$\Delta T 1$} & $\mu$ & -0.009 & -0.009 & -0.008 & -0.007 \\
\hline & $\sigma$ & 0.011 & 0.009 & 0.007 & 0.006 \\
\hline \multirow[t]{2}{*}{$\Delta T 2$} & $\mu$ & -0.006 & -0.006 & -0.006 & -0.006 \\
\hline & $\sigma$ & $\begin{array}{c}9.5657 \\
\text { e- } 04\end{array}$ & $\begin{array}{c}7.5452 \\
\text { e- } 04\end{array}$ & $\begin{array}{c}5.4490 \\
\text { e- } 04\end{array}$ & $\begin{array}{c}3.7603 \\
e-04\end{array}$ \\
\hline
\end{tabular}

Table 4 Statistics of delta K, delta T1 and delta T2 for case 2

Case 3

Assuming there are visual perception noise and the neuromuscular noise in the human controller model, we want to know if the Kalman filter can handle the neuromuscular noise. The estimation results are plot in figure 22 and tabulate in table 5. From table 5 and figure 22, it can be seen that the estimation results also converge to the parameters preset in the human controller very quickly. This indicates that the Kalman filter can handle the neuromuscular noise without a priori knowledge about it.

\begin{tabular}{|c|l|l|l|l|l|}
\hline \multicolumn{2}{|c|}{} & $\mathrm{R}=0.1$ & $\mathrm{R}=1$ & $\mathrm{R}=10$ & $\mathrm{R}=100$ \\
\hline$\Delta K$ & $\mu$ & 0.3616 & 0.3553 & 0.3383 & 0.3178 \\
\hline & $\sigma$ & 0.2093 & 0.1706 & 0.1397 & 0.1189 \\
\hline \multirow{2}{*}{$\Delta T 1$} & $\mu$ & 0.0149 & 0.0137 & 0.0108 & 0.0076 \\
\hline & $\sigma$ & 0.0315 & 0.0252 & 0.0201 & 0.0167 \\
\hline \multirow{2}{*}{$\Delta T 2$} & $\mu$ & -0.008 & -0.008 & -0.008 & -0.008 \\
\hline & $\sigma$ & 0.0025 & 0.0019 & 0.0013 & $\begin{array}{c}9.3372 \\
\text { e-04 }\end{array}$ \\
\hline
\end{tabular}

Table 5 Statistics of delta K, delta T1 \& delta T2 for case 3 

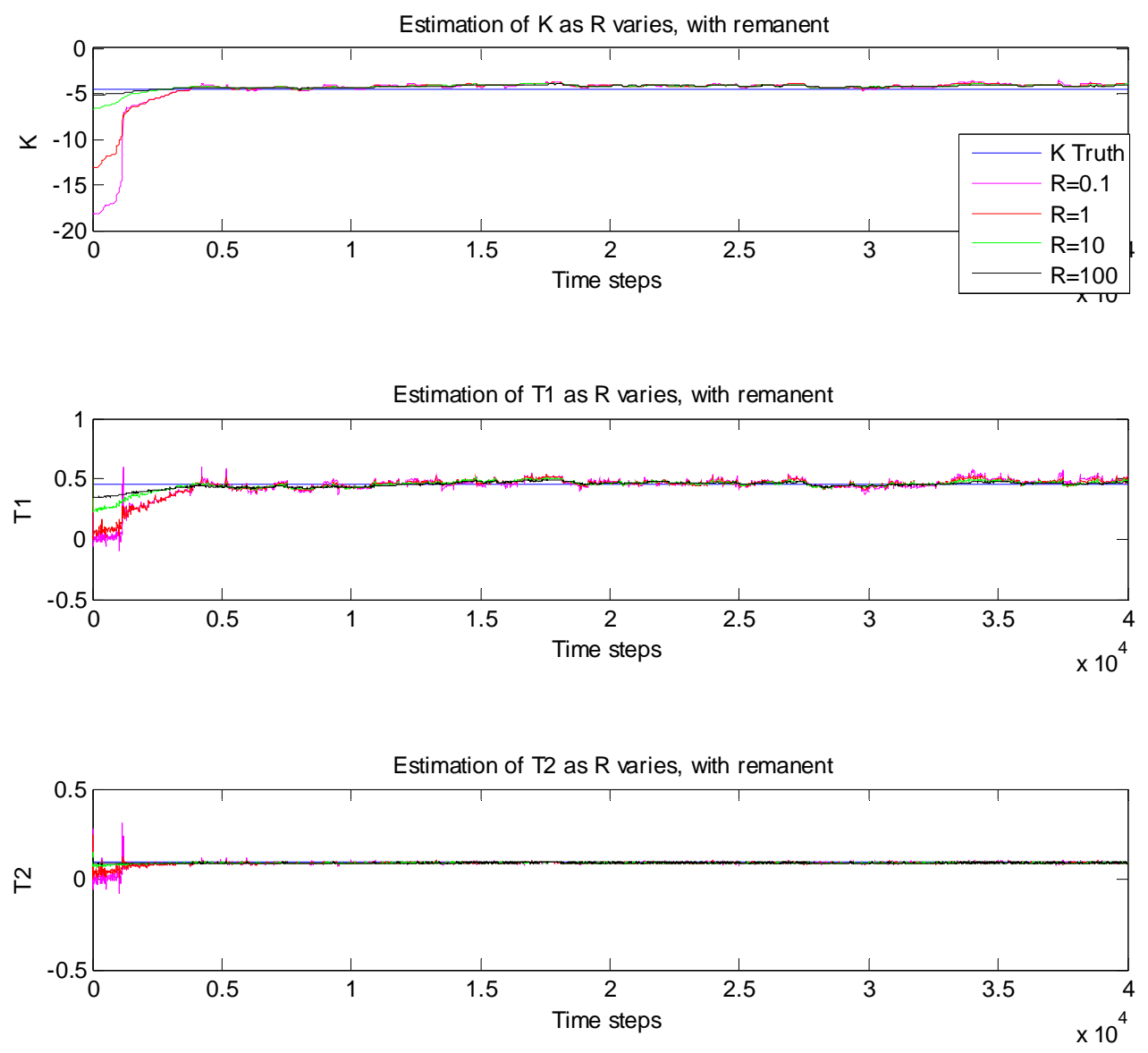

Figure 22 Estimations of $\mathrm{K}, \mathrm{T} 1$ \& T2 as R varies, with remnant noise

\section{Case 4}

So far, the delay present in the human controller model has not yet been addressed. In this case, different values of time delay are chosen and set in the model, assuming the delay is known perfectly, the data to the Kalman filter are shifted according to the delay value beforehand. The estimation results, as can be seen from figure 23 and table 6 , indicates that the Kalman filter can estimate $K, T_{1}$, and $T_{2}$ reasonably well. 

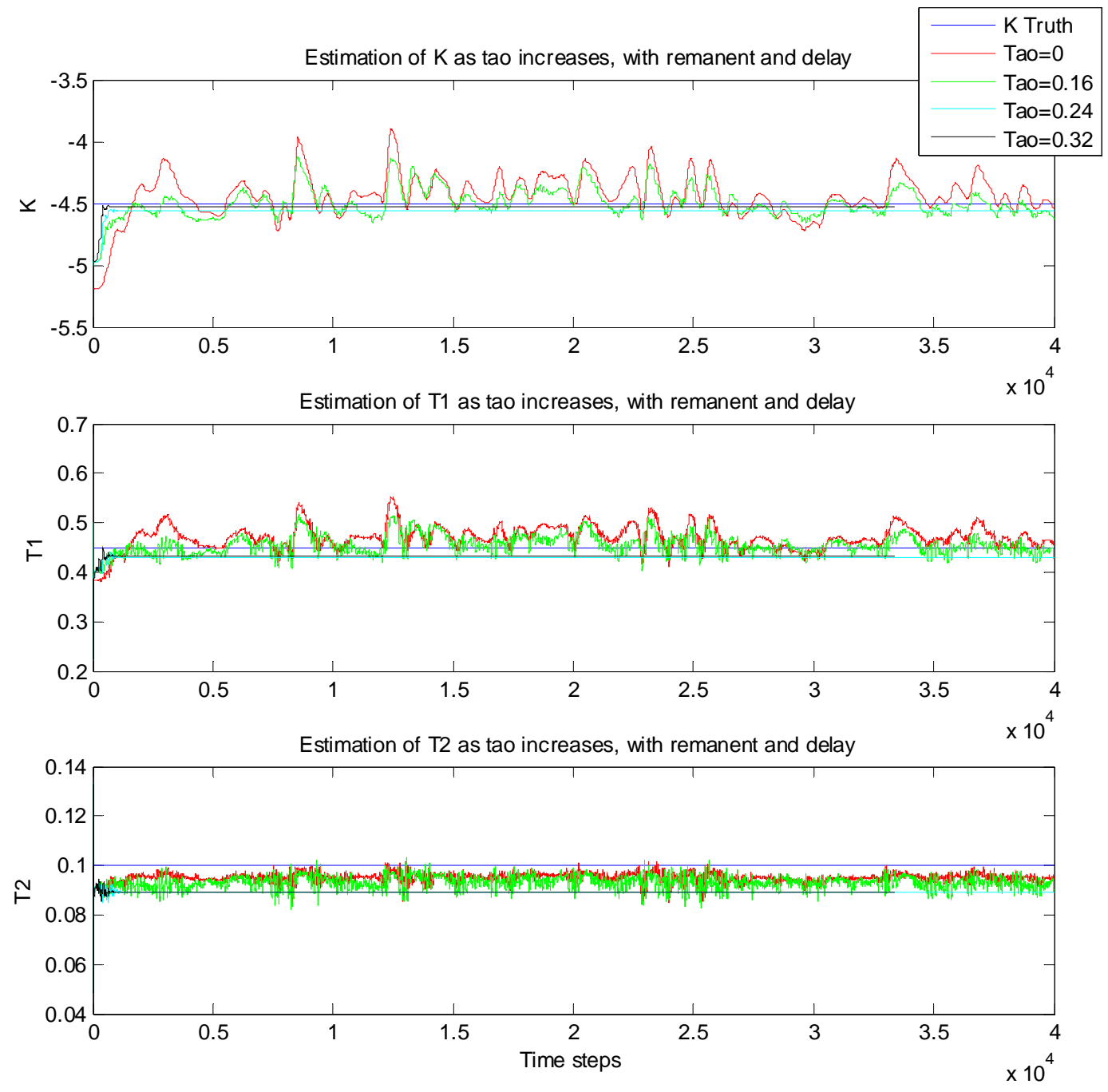

Figure 23 Estimations of K, T1 \& T2 as tao increases, with remnant and delay

\begin{tabular}{|c|c|c|c|c|c|}
\hline & $\mathrm{R}=0.1$ & $R=1$ & $R=10$ & $R=100$ \\
\hline \multirow[t]{2}{*}{$\Delta K$} & $\mu$ & 0.0816 & 0.0211 & -0.059 & -0.052 \\
\hline & $\sigma$ & 0.1423 & 0.1050 & $\begin{array}{c}2.8 \mathrm{e}- \\
13\end{array}$ & $\begin{array}{c}3.2 \mathrm{e}- \\
13\end{array}$ \\
\hline \multirow[t]{2}{*}{$\Delta T 1$} & $\mu$ & 0.0216 & 0.0069 & -0.019 & -0.017 \\
\hline & $\sigma$ & 0.0213 & 0.0184 & $\begin{array}{c}4.2 \mathrm{e}- \\
13\end{array}$ & $\begin{array}{c}3.6 \mathrm{e}- \\
13\end{array}$ \\
\hline \multirow[t]{2}{*}{$\Delta T 2$} & $\mu$ & -0.005 & -0.006 & -0.010 & -0.011 \\
\hline & $\sigma$ & 0.0017 & 0.0022 & $\begin{array}{c}4.8 \mathrm{e}- \\
13\end{array}$ & $\begin{array}{c}3.8 \mathrm{e}- \\
13\end{array}$ \\
\hline
\end{tabular}

Table 6 Statistics of delta K, delta T1 \& delta T2 for case 4 


\section{Case 5}

What if the delay is not perfectly known? In this case, the data can still be shifted by some values of time delay but with uncertainty. The estimation results are plotted in figure 25 . As can be seen from figure 25 , the estimation results do not converge to the parameters set in the model even if the data were only mismatch by a single simulation time step, which is $0.01 \mathrm{~s}$.
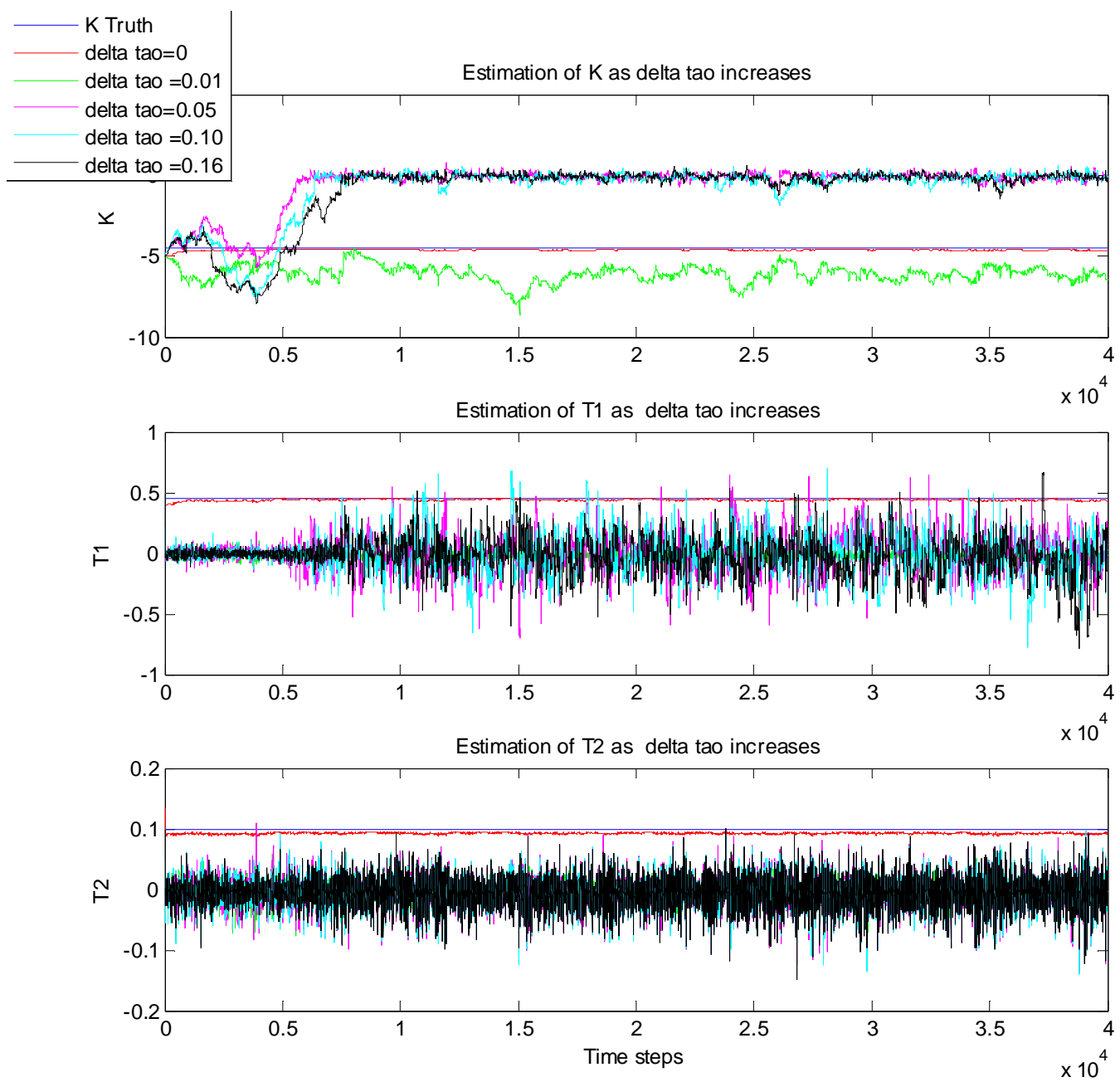

Figure 24 Estimations of K, T1 \& T2 as delta tao increases, with remnant and delay 


\begin{tabular}{|c|c|c|c|c|c|c|}
\hline & & $\begin{array}{l}\Delta \tau \\
=0\end{array}$ & $\begin{array}{c}\Delta \tau= \\
0.01\end{array}$ & $\begin{array}{l}\Delta \tau= \\
0.05\end{array}$ & $\begin{array}{l}\Delta \tau= \\
0.10\end{array}$ & $\begin{array}{l}\Delta \tau= \\
0.16\end{array}$ \\
\hline \multirow[t]{2}{*}{$\Delta K$} & $\mu$ & -0.10 & -1.59 & 4.388 & 4.248 & 4.190 \\
\hline & $\sigma$ & 0.018 & 0.518 & 0.272 & 0.549 & 0.789 \\
\hline \multirow[t]{2}{*}{$\Delta T 1$} & $\mu$ & $\begin{array}{c}- \\
0.009\end{array}$ & $\begin{array}{c}- \\
0.467\end{array}$ & $\begin{array}{c}- \\
0.456\end{array}$ & 0. & $\begin{array}{c}- \\
0.474\end{array}$ \\
\hline & $\sigma$ & 0.005 & 0.013 & 0.140 & 0.142 & 0.143 \\
\hline \multirow[t]{2}{*}{$\Delta T 2$} & $\mu$ & $\begin{array}{c}- \\
0.007\end{array}$ & $\begin{array}{c}- \\
0.103\end{array}$ & $\begin{array}{c}- \\
0.104\end{array}$ & $\begin{array}{c}- \\
0.104\end{array}$ & $\begin{array}{c}- \\
0.104\end{array}$ \\
\hline & $\sigma$ & 0.001 & 0.078 & 0.015 & 0.014 & 0.014 \\
\hline
\end{tabular}

Table 7 Statistics of delta K, delta T1 \& delta T2 for case 5

\section{Case 6}

Finally, the parameters in the human controller model namely $K, T_{1}$, and $T_{2}$ are assumed to be timevarying. Also, sensory noises and delay are also assumed to be present in this case. This is the most realistic scenario of all cases that have been discussed in this section. The estimation results are plotted in figure 24 and tabulated in table 7 . From them, it can be seen that the filter can estimate $K, T_{1}$, and $T_{2}$ quite well.

\begin{tabular}{|l|l|l|l|}
\hline & & TAO $=0$ & TAO $=0.16$ \\
\hline$\Delta K$ & $\mu$ & 0.1020 & 0.1141 \\
\hline \multirow{2}{*}{$\Delta T 1$} & $\sigma$ & 0.2760 & 0.1914 \\
\hline \multirow{2}{*}{$\Delta T 2$} & $\mu$ & 0.0139 & 0.0175 \\
\hline & $\sigma$ & 0.0496 & 0.0321 \\
\hline & $\mu$ & -0.0073 & -0.0066 \\
\hline & $\sigma$ & 0.0062 & 0.0041 \\
\hline
\end{tabular}

Table 8 Statistics of delta K, delta T1 \& delta T2 for case 6 

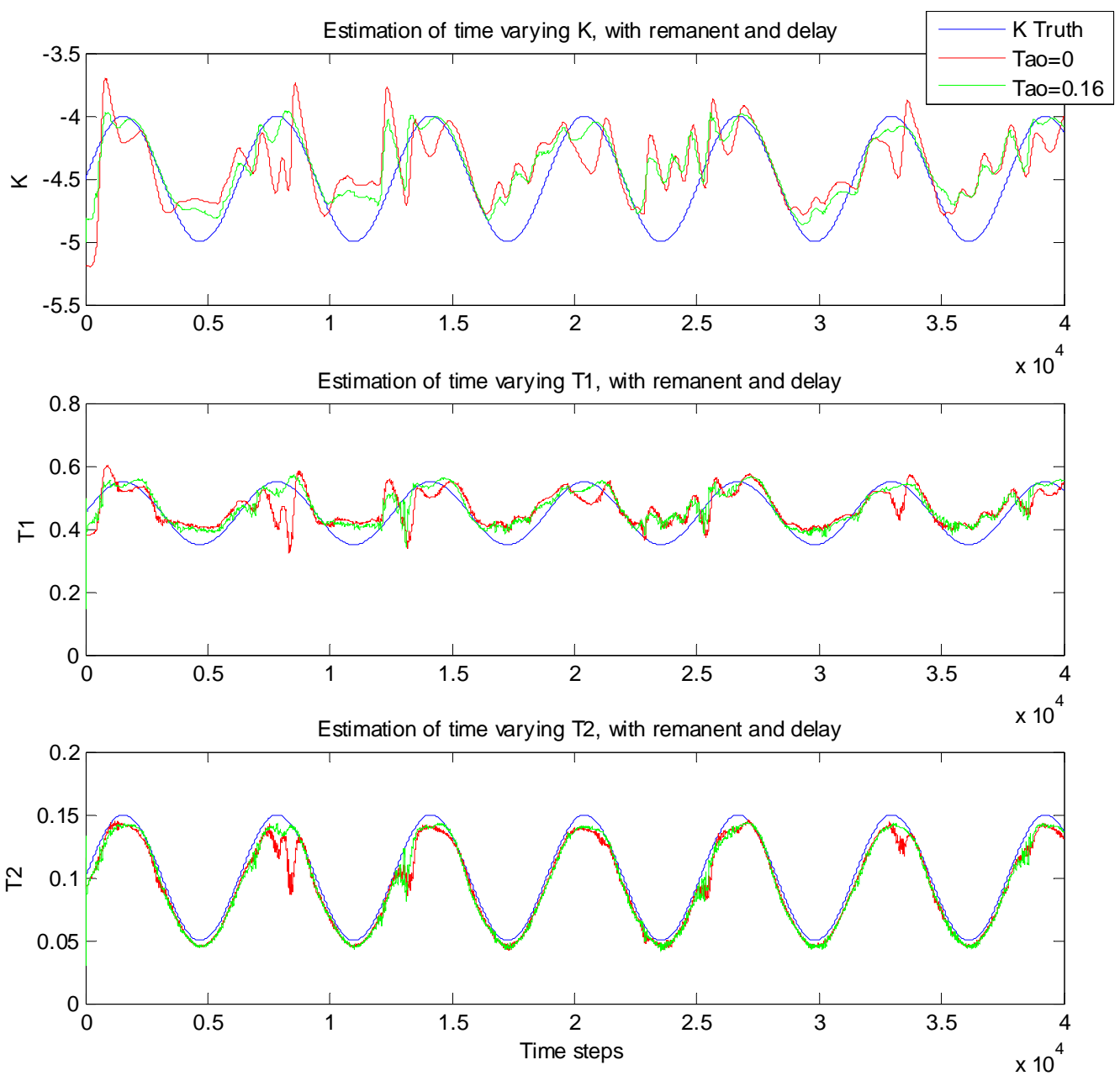

Figure 25 Estimations of $\mathrm{K}, \mathrm{T} 1$ \& T2 when they vary with time

In conclusion, the Kalman filter developed in this section can do a reasonably good job in estimating the parameters in the human controller model with only two assumptions. The first one is that, the visual perception noise that the theta angle is subject to has to be known with high certainty. Secondly, the amount of delay in the human controller model has to be known so the data can be shifted accordingly before it is used to do the estimation. 


\subsection{Estimation Results from Experimental Data}

This section presents the estimation results from the experimental data using the Kalman filter developed and verified in the section 6.1. Figure 26 is the block diagram of the Matlab scheme used for collecting and processing the experimental data. Figure $27,28,29 \& 30$ present the estimation results from four different subjects using the Kalman filter aforementioned. To assess the performance of the estimation, the estimated $K, T_{1}$, and $T_{2}$ were used to stabilize the inverted pendulum simulation in Simulink because we don't have the a priori truth of $K, T_{1}$, and $T_{2}$. Unfortunately, the estimated $K, T_{1}$, and $T_{2}$ cannot always stabilize the inverted pendulum even when the experimental data shows the pendulum is, in fact, in balance. This implies that the estimation results from the experimental data need further interpretation.

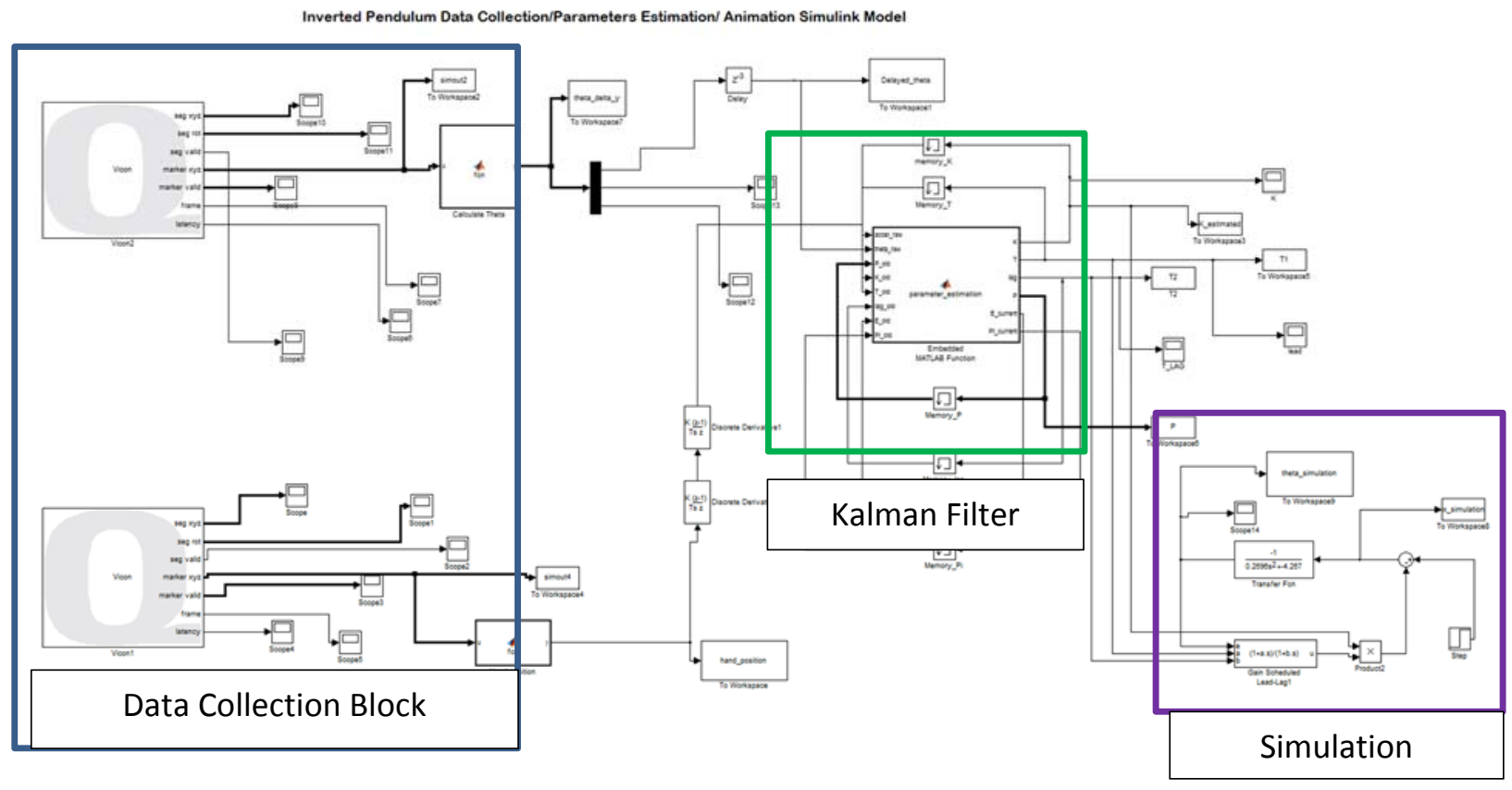

Figure 26 Matlab Simulation Model 

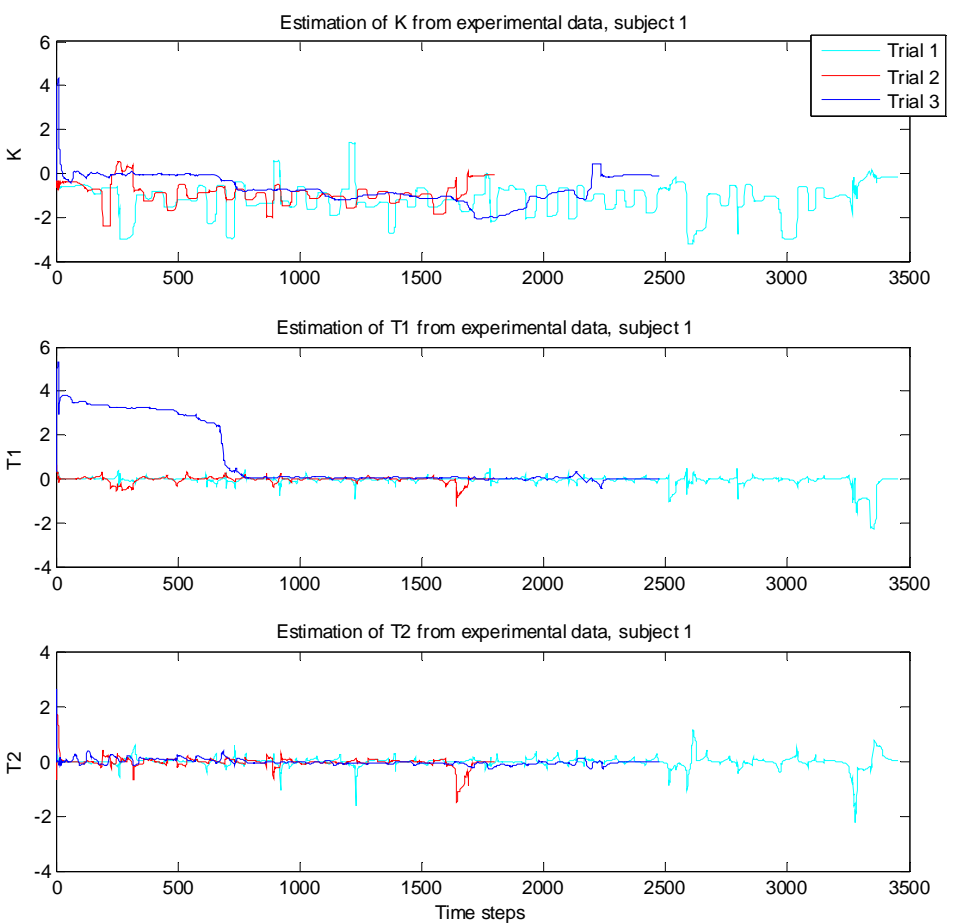

Figure 27 Estimations of K, T1 \& T2 from experimental data, subject 1
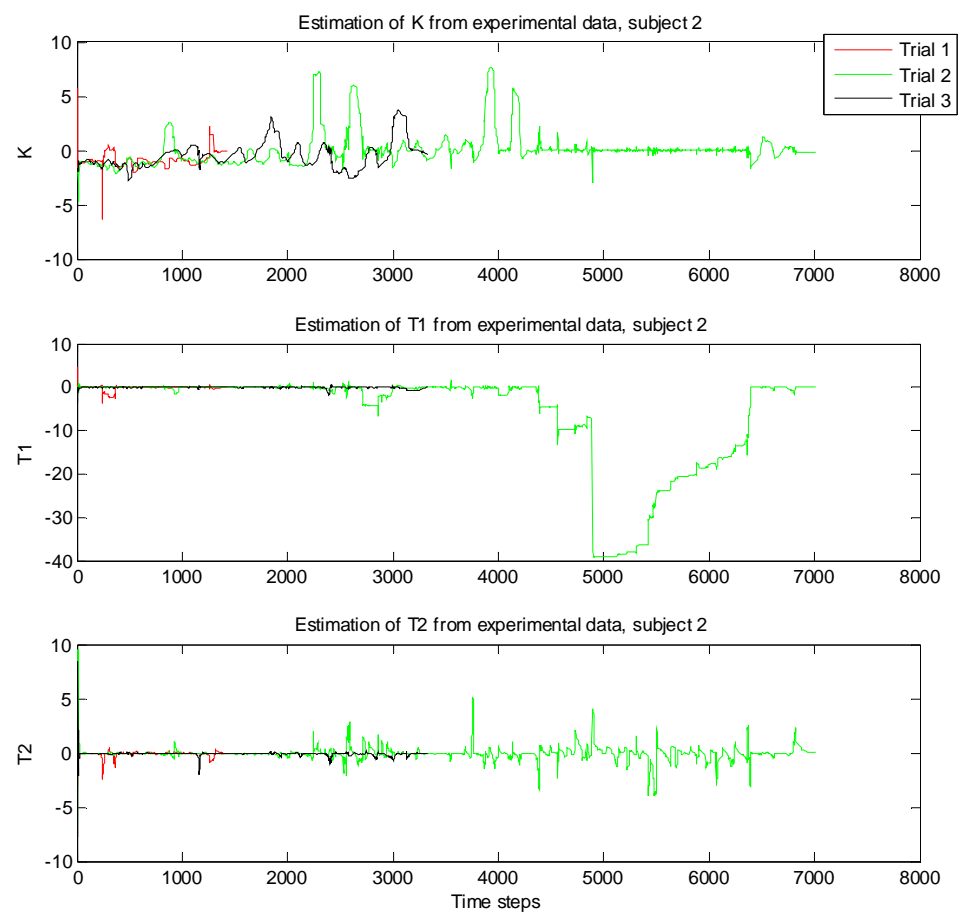

Figure 28 Estimations of K, T1 \& T2 from experimental data, subject 2 

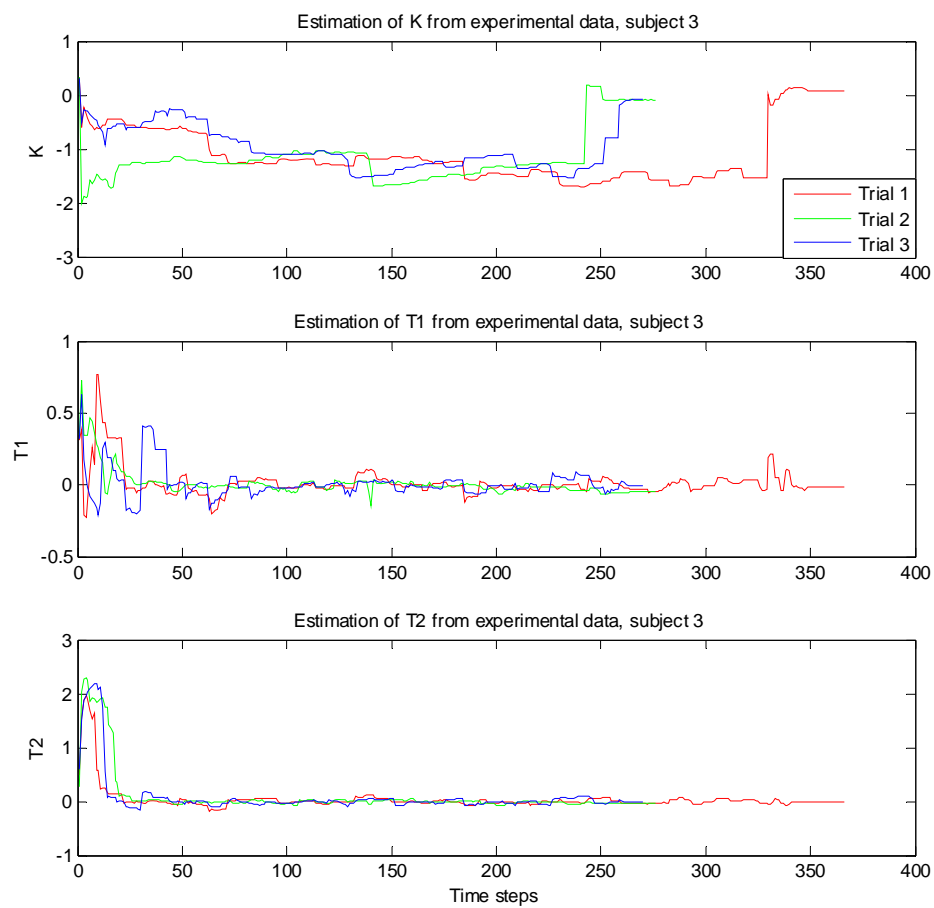

Figure 29 Estimations of K, T1 \& T2 from experimental data, subject 3
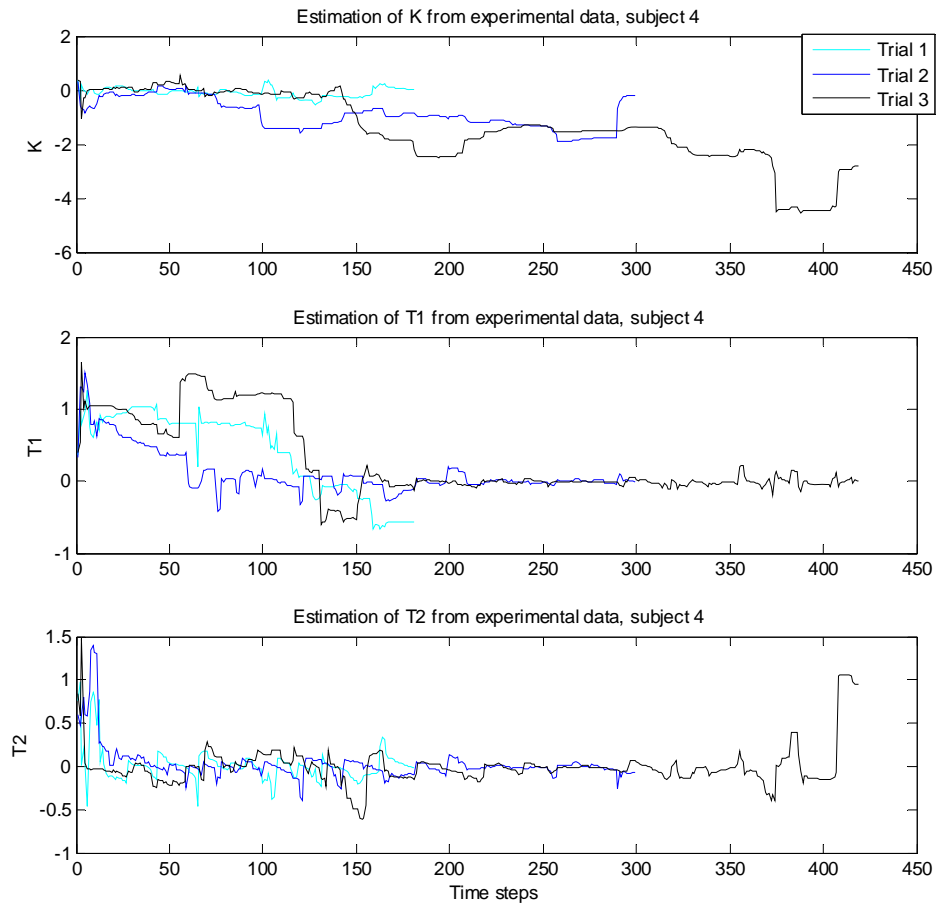

Figure 30 Estimations of $K, T 1 \&$ T2 from experimental data, subject 4 


\section{CHAPTER 7 CONCLUSIONS}

This study attempted to quantitatively study the characteristics of human manual control under a singleaxil compensatory task, e.g. balancing an inverted pendulum or controlling the pitch angle of an airplane. To that end, a single-axial inverted pendulum setup was built. A mathematical model of the closed-loop human inverted pendulum system was obtained. Subjects were asked to balance the inverted pendulum by sliding a base upon which the inverted pendulum was mounted. Stability analysis of closed-loop human inverted pendulum was performed. The stability conditions for the human controller are greatly influenced by the delay present in the human subject. A Kalman filter was developed for estimating the parameters in the human controller. Simulation results show that the estimator can accurately estimate the parameters set in human controller's model. When the estimator is used to estimate the parameters using the experimental data, the estimated parameters do not always stabilize the pendulum. This could have resulted from three possible causes. The measured angle deflection of the inverted pendulum is corrupted by an unknown noise; this noise cannot be handled by the filter, but has to be compensated before the data is fed to the estimator. Secondly, it turns out that the delay in the closed-loop does not only greatly affect the stability of the system but also greatly affects the estimation results. From, the simulation, it is shown that if the delay is perfectly offset in the data, the estimation results converge very well to the parameters set in the model. But as long as there is a slight mismatch between the data, i.e., the delay is not perfectly known, the estimation results become very different from the parameters set in the model. In real life scenario, the delay present in the human controller is hard to obtain and likely time-varying. Thirdly, the parameters in human controller model are likely time varying as well. These possible factors that could affect the performance of the estimator were thoroughly discussed and tested using the simulation developed. The findings from the study are summarized below. 
1. $\tau_{1}^{2}<\frac{-2 a}{b}$, the maximum delay allowable in the controller is determined by the pendulum's length and weight.

2. The estimator can handle the neuromuscular noise but not the noise in the visual perception system, this is likely due to formulation of the Kalman filter.

3. The Kalman filter can estimate the parameters in the human controller model even when the parameters are time-varying.

4. The Kalman filter can estimate the parameters in the human controller model when there is timevarying delay present in the system.

As can be seen from chapter six, the estimation results from the experimental data need further interpretation. Time varying and subject dependent nature of the amount of delay or the unknown visual perception noise in the human controller are likely the reasons the estimated parameters from the experimental data cannot stabilize the pendulum. Thus, future directions of the study include finding ways to handle the unknown visual perception noise present in the human controller and to estimate the amount of delay present in the human controller. Additionally, different models of the human controller, such as a dynamic inversion model of the human controller, can be tried out to make sense of the estimation from the experimental data. 


\section{References}

[1] B. Widrow and F. W. Smith, "Pattern-recognizing control systems," Computer and Information Sciences (COINS) Proceedings, 1964.

[2] B. Widrow, "The original adaptive neural net broom-balancer," in IEEE International Symposium on Circuits and Systems, 1987.

[3] V. V. Tolat and B. Widrow, "An adaptive'broom balancer'with visual inputs," in Neural Networks, 1988., IEEE International Conference on, 1988, pp. 641-647.

[4] C. W. Anderson, "Learning to control an inverted pendulum using neural networks," Control Systems Magazine, IEEE, vol. 9, pp. 31-37, 1989.

[5] A. P. Wieland, "Evolving neural network controllers for unstable systems," in Neural Networks, 1991., IJCNN-91-Seattle International Joint Conference on, 1991, pp. 667-673.

[6] S. Jung and S. S. Kim, "Control experiment of a wheel-driven mobile inverted pendulum using neural network," Control Systems Technology, IEEE Transactions on, vol. 16, pp. 297-303, 2008.

[7] M. E. Magana and F. Holzapfel, "Fuzzy-logic control of an inverted pendulum with vision feedback," Education, IEEE Transactions on, vol. 41, pp. 165-170, 1998.

[8] L.-X. Wang, "Stable adaptive fuzzy controllers with application to inverted pendulum tracking," Systems, Man, and Cybernetics, Part B: Cybernetics, IEEE Transactions on, vol. 26, pp. 677-691, 1996.

[9] M. I. El-Hawwary, A.-L. Elshafei, H. M. Emara, and H. A. A. Fattah, "Adaptive fuzzy control of the inverted pendulum problem," Control Systems Technology, IEEE Transactions on, vol. 14, pp. 1135-1144, 2006.

[10] M. Bugeja, "Non-linear swing-up and stabilizing control of an inverted pendulum system," in EUROCON 2003. Computer as a Tool. The IEEE Region 8, 2003, pp. 437-441.

[11] K. Furuta, M. Yamakita, and S. Kobayashi, "Swing-up control of inverted pendulum using pseudostate feedback," Proceedings of the Institution of Mechanical Engineers, Part I: Journal of Systems and Control Engineering, vol. 206, pp. 263-269, 1992.

[12] N. Muskinja and B. Tovornik, "Swinging up and stabilization of a real inverted pendulum," Industrial Electronics, IEEE Transactions on, vol. 53, pp. 631-639, 2006.

[13] S. Yasunobu and M. Mori, "Swing up fuzzy controller for inverted pendulum based on a human control strategy," in Fuzzy Systems, 1997., Proceedings of the Sixth IEEE International Conference on, 1997, pp. 1621-1625.

[14] S. Kajita and K. Tani, "Study of dynamic biped locomotion on rugged terrain-derivation and application of the linear inverted pendulum mode," in Robotics and Automation, 1991. Proceedings., 1991 IEEE International Conference on, 1991, pp. 1405-1411.

[15] S. Kajita and K. Tani, "Experimental study of biped dynamic walking in the linear inverted pendulum mode," in Robotics and Automation, 1995. Proceedings., 1995 IEEE International Conference on, 1995, pp. 2885-2891.

[16] S. Kajita, F. Kanehiro, K. Kaneko, K. Fujiwara, K. Harada, K. Yokoi, et al., "Biped walking pattern generation by using preview control of zero-moment point," in Robotics and Automation, 2003. Proceedings. ICRA'03. IEEE International Conference on, 2003, pp. 1620-1626.

[17] S. Kajita, M. Morisawa, K. Miura, S. Nakaoka, K. Harada, K. Kaneko, et al., "Biped walking stabilization based on linear inverted pendulum tracking," in Intelligent Robots and Systems (IROS), 2010 IEEE/RSJ International Conference on, 2010, pp. 4489-4496.

[18] S. Kajita, F. Kanehiro, K. Kaneko, K. Yokoi, and H. Hirukawa, "The 3D Linear Inverted Pendulum Mode: A simple modeling for a biped walking pattern generation," in Intelligent Robots and Systems, 2001. Proceedings. 2001 IEEE/RSJ International Conference on, 2001, pp. 239-246. 
[19] J. H. Park and K. D. Kim, "Biped robot walking using gravity-compensated inverted pendulum mode and computed torque control," in Robotics and Automation, 1998. Proceedings. 1998 IEEE International Conference on, 1998, pp. 3528-3533.

[20] A. D. Kuo, "The six determinants of gait and the inverted pendulum analogy: A dynamic walking perspective," Human movement science, vol. 26, pp. 617-656, 2007.

[21] T. Sugihara, Y. Nakamura, and H. Inoue, "Real-time humanoid motion generation through ZMP manipulation based on inverted pendulum control," in Robotics and Automation, 2002. Proceedings. ICRA'02. IEEE International Conference on, 2002, pp. 1404-1409.

[22] W. H. Gage, D. A. Winter, J. S. Frank, and A. L. Adkin, "Kinematic and kinetic validity of the inverted pendulum model in quiet standing," Gait \& posture, vol. 19, pp. 124-132, 2004.

[23] D. A. Winter, "Human balance and posture control during standing and walking," Gait \& posture, vol. 3, pp. 193-214, 1995.

[24] N. P. Reeves, K. S. Narendra, and J. Cholewicki, "Spine stability: lessons from balancing a stick," Clinical Biomechanics, vol. 26, pp. 325-330, 2011.

[25] G. W. Housner, "The behavior of inverted pendulum structures during earthquakes," Bulletin of the seismological society of America, vol. 53, pp. 403-417, 1963.

[26] A. T. Bahill, M. R. Clark, and L. Stark, "The main sequence, a tool for studying human eye movements," Mathematical Biosciences, vol. 24, pp. 191-204, 1975.

[27] S. Lisberger and L. Westbrook, "Properties of visual inputs that initiate horizontal smooth pursuit eye movements in monkeys," The Journal of neuroscience, vol. 5, pp. 1662-1673, 1985.

[28] J. Schlag and M. Schlag-Rey, "Through the eye, slowly; Delays and localization errors in the visual system," Nature Reviews Neuroscience, vol. 3, pp. 191-191, 2002.

[29] A. B. Watson and A. J. Ahumada Jr, "Model of human visual-motion sensing," JOSA A, vol. 2, pp. 322-341, 1985.

[30] M. Kawato, "Internal models for motor control and trajectory planning," Current opinion in neurobiology, vol. 9, pp. 718-727, 1999.

[31] D. M. Wolpert, Z. Ghahramani, and M. I. Jordan, "An internal model for sensorimotor integration," Science-AAAS-Weekly Paper Edition, vol. 269, pp. 1880-1882, 1995.

[32] J. I. Elkind, "Characteristics of simple manual control systems," Massachusetts Institute of Technology, 1956.

[33] L. Young, "On adaptive manual control," Ergonomics, vol. 12, pp. 635-674, 1969.

[34] D. T. McRuer and H. R. Jex, "A review of quasi-linear pilot models," Human Factors in Electronics, IEEE Transactions on, pp. 231-249, 1967.

[35] R. E. Magdaleno and D. T. Mc Ruer, "Experimental validation and analytical elaboration for models of the pilot's neuromuscular subsystem in tracking tasks," 1971.

[36] G. B. Greg Welch. (July 24, 2006, An introduction to the Kalman filter. Available: https://www.cs.unc.edu/ welch/media/pdf/kalman intro.pdf

[37] R. E. Kalman, "A new approach to linear filtering and prediction problems," Journal of Fluids Engineering, vol. 82, pp. 35-45, 1960.

[38] Z. Chen, "Bayesian filtering: From Kalman filters to particle filters, and beyond," Statistics, vol. 182, pp. 1-69, 2003.

[39] S. J. Julier and J. K. Uhlmann, "Unscented filtering and nonlinear estimation," Proceedings of the IEEE, vol. 92, pp. 401-422, 2004.

[40] P. M. Zaal and B. T. Sweet, "Estimation of time-varying pilot model parameters," in AIAA Modeling and Simulation Technologies Conference, Portland, OR (8-11 August 2011), 2011.

[41] K. Ogata and Y. Yang, "Modern control engineering," 1970. 


\section{Appendix one Derivation of the EOM's of the Inverted Pendulum Setup}

The schematic of the inverted pendulum-cart system is shown in figure 3 . The cart can only slide on a single axis rail. The control force $u$ is applied to the cart by the human subject's hand. Assume that the center of gravity of the inverted pendulum is at its geometric center. In this appendix, the Equations of Motion are derived using the LaGrange's method. The EOM's is then linearized by using the small angle approximation. Finally, the transfer functions are obtained by taking the Laplace transform of the linearized EOM's.

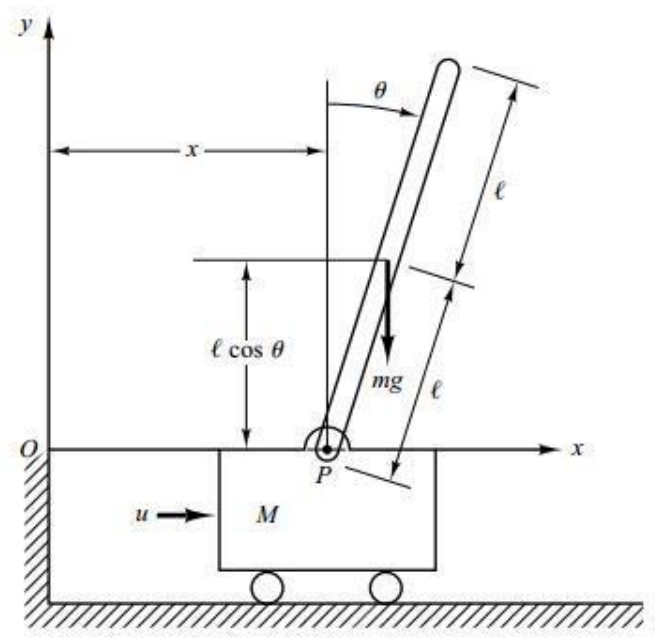

Figure 31 Schematic of the Inverted Pendulum-Cart System

\subsection{Generalized Coordinate System}

In order to apply the LaGrange's method to the system, we need to first choose the generalized coordinates. Using the generalized coordinates, we then can determine the equations for the generalized forces, kinetic and potential energies etc. The inverted pendulum system has two degrees of freedom and therefore can be fully represented by two generalized coordinates. The generalized coordinates $q_{j}$ are chosen as the horizontal displacement of the sliding cart $x$ and the angular displacement of the inverted pendulum $\theta$. 


$$
q_{j}: x, \theta, j=1,2
$$

The positive direction of $x$ is to the right, measured from the left end of the rail and the positive direction of $\theta$ is clockwise, measured from the upright position.

\subsection{Generalized Coordinate Forces}

The generalized forces $Q_{j}$ can be derived from the non-conservative work. The non-conservative force in our case is the input force $u(t)$, if we choose to ignore the frictions between the sliding base and the rail, and the frictions of the pivoting joint. The generalized force is given by

$$
Q_{1}=u(t)
$$

\subsection{Kinetic and Potential Energy Functions}

The kinetic energy of the inverted pendulum cart system is given by

$$
T=\frac{1}{2} M v_{M}^{2}+\frac{1}{2} m v_{m}^{2}+\frac{1}{2} I_{m} \dot{\theta}^{2}
$$

Where, $I_{m}$ is the moment of inertial of the inverted pendulum around the pivoting point, and $v_{M}$ is the velocity of the sliding base, $v_{m}$ is the velocity of the pendulum's center of mass. The velocities can be related to the generalized coordinates specified above by the following two equations.

$$
\begin{gathered}
v_{M}=\dot{x} \\
v_{m}=\dot{x}+\dot{\theta} l \cos \theta
\end{gathered}
$$

Substituting equations (48) and (49) into equations (47) yields 


$$
T=\frac{1}{2} M \dot{x}^{2}+\frac{1}{2} m\left[\dot{x}^{2}+2(l \dot{\theta} \cos \theta) \dot{x}+l^{2} \dot{\theta}^{2} \cos ^{2} \theta\right]+\frac{1}{2} I_{m} \dot{\theta}^{2}
$$

This, upon simplification, becomes

$$
T=\frac{1}{2}(m+M) \dot{x}^{2}+m l \dot{x} \dot{\theta} \cos \theta+\frac{1}{2} m l^{2} \dot{\theta}^{2} \cos ^{2} \theta+\frac{1}{2} I_{m} \dot{\theta}^{2}
$$

The potential energy of the system is determined by the height of the center of mass of the inverted pendulum, given by

$$
U=m g l \cos \theta
$$

\subsection{Lagrangian}

The Lagrange's equation is given by

$$
L=T-U
$$

Using equations (51) and (52), the Lagrangian can be written as

$$
L=\frac{1}{2}(m+M) \dot{x}^{2}+m l \dot{x} \dot{\theta} \cos \theta+\frac{1}{2} m l^{2} \dot{\theta}^{2} \cos ^{2} \theta+\frac{1}{2} I_{m} \dot{\theta}^{2}-m g l \cos \theta
$$

\subsection{Lagrange's Equation}

Lagrange's equation of the generalized coordinate is given by the following formula

$$
\frac{d}{d t}\left(\frac{\partial L}{\partial \dot{q}_{j}}\right)-\frac{\partial L}{\partial q_{j}}=Q_{j}
$$

Forq $=x$, the equation becomes 


$$
\frac{d}{d t}\left(\frac{\partial L}{\partial \dot{x}}\right)-\frac{\partial L}{\partial x}=u(\mathrm{t})
$$

The partial derivative of $L$ with respect to $\dot{x}$ is

$$
\frac{\partial L}{\partial \dot{x}}=m \dot{x}+M \dot{x}+m(l \dot{\theta} \cos \theta)
$$

The derivative of $\frac{\partial L}{\partial \dot{x}}$ with respect to time $t$ is

$$
\frac{d}{d t}\left(\frac{\partial L}{\partial \dot{x}}\right)=m \ddot{x}+M \ddot{x}+m\left(l \ddot{\theta} \cos \theta-l \dot{\theta}^{2} \sin \theta\right)
$$

The derivative of $L$ with respect to $x$ is zero.

$$
\frac{\partial L}{\partial x}=0
$$

Substituting (58) and (59) into (56), we get

$$
m \ddot{x}+M \ddot{x}+m l \ddot{\theta} \cos \theta-m l \dot{\theta}^{2} \sin \theta=u(\mathrm{t})
$$

For $q=\theta$, the lagrange's equation is

$$
\frac{d}{d t}\left(\frac{\partial L}{\partial \dot{\theta}}\right)-\frac{\partial L}{\partial \theta}=0
$$

The partial derivative of $L$ with respect to $\dot{\theta}$ is

$$
\frac{\partial L}{\partial \dot{\theta}}=m l \dot{x} \cos \theta+m l^{2} \dot{\theta} \cos ^{2} \theta+I_{m} \dot{\theta}
$$

The derivative of partial T with respect to time is 


$$
\frac{d}{d t}\left(\frac{\partial L}{\partial \dot{\theta}}\right)=m l \ddot{x} \cos \theta-m l \dot{x} \dot{\theta} \sin \theta+m l^{2} \ddot{\theta} \cos ^{2} \theta-m l^{2} \dot{\theta}^{2} \sin 2 \theta+I_{m} \ddot{\theta}
$$

The derivative of $L$ with respect to $\theta$ is given by

$$
\frac{\partial L}{\partial \theta}=-m l \dot{x} \dot{\theta} \sin \theta+m g l \sin \theta
$$

Substituting (63) and (64) into (61), we get

$$
m l \ddot{x} \cos \theta+m l^{2} \ddot{\theta} \cos ^{2} \theta-m l^{2} \dot{\theta}^{2} \sin 2 \theta+I_{m} \ddot{\theta}-m g l \sin \theta=0
$$

Together, equations (16) and (21) comprise the EOM's of the inverted pendulum system.

\subsection{Linearization and Transfer Function Generation}

The nonlinear EOM's (60) and (65) of the system are very hard to deal with. Fortunately, the behavior of the inverted pendulum when it's balanced near the upright position can be well modeled by the linearized EOM's. By the coordinates we chose and the origin of the coordinate system, $\theta$ is very small when the pendulum is near the upright position. Thus we can apply the small angle approximation where,

$$
\begin{gathered}
\sin \theta \approx \theta \\
\cos \theta \approx 1 \\
\dot{\theta} \approx 0
\end{gathered}
$$

To the EOM'S we just derived and equations (60) and (65) can be simplified as

$$
m \ddot{x}+M \ddot{x}+m l \ddot{\theta}=u(\mathrm{t})
$$




$$
m l \ddot{x}+\left(m l^{2}+I_{m}\right) \ddot{\theta}-m g l \theta=0
$$

